\title{
Climbing the Drug Staircase: A Bayesian Analysis of the Initiation of Hard Drug Use
}

\author{
Anne Line Bretteville-Jensen \\ Norwegian Institute for Alcohol and Drug Research (SIRUS) \\ LIANA JACOBI* \\ Department of Economics, University of Melbourne
}

September 2009

\begin{abstract}
SUMMARY
Since empirical studies have shown that cannabis users are much more likely to initiate hard drug use, a causal linkage has been suggested ("gateway hypothesis"). However, individual differences in proneness and accessibility to drugs provide alternative non-causal explanations for the observed drug use pattern. We propose a Bayesian estimation and predictive framework to analyze the effects and relative importance of previous cannabis use, proneness and accessibility factors on hard drug initiation and to explore possible policy implications. We employ a novel model specification, motivated by four gateway transmission channels, to analyze data from a recent Norwegian survey of young adults.
\end{abstract}

Key words: Bayesian Prior-Posterior Analysis, Bayesian Predictive Analysis, Bivariate Probit, Cannabis Gateway, Cannabis Use, Hard Drug use.

${ }^{*}$ Correspondence to: Liana Jacobi, University of Melbourne, Department of Economics, 5th floor Economics and Commerce Building, 3010 Victoria, Australia; email: ljacobi@unimelb.edu.au; phone: +61 38344 5289; fax +61383446899. 


\section{Introduction}

Cannabis is the most widely-used illicit drug in Europe, Australia and North America. Global estimates suggest that currently there are about 160 million cannabis users and in many countries prevalence numbers have risen over the last 10 years (UN, 2007). An interesting question is whether this widespread cannabis use will also lead to an increase in the number of hard drug users. The question emerges because one of the most robust findings in the epidemiology of illicit drug use is the initiation pattern, where most illicit drug users have started with legal drugs like alcohol and cigarettes before proceeding to cannabis and subsequently, if further involvement with illegal drugs, to hard drugs (Kandel, 2002). For example, very few hard drug users go straight to heroin without previously having used several other drugs and for many cannabis was the first illegal drug used. Kandel (1975), employing a US sample, was the first to point out this "staircase" but a similar pattern has been reported in many subsequent studies of drug initiation (Kandel, 2002).

The observed staircase pattern of illegal drug initiation has led to the so called gateway hypothesis. It states that the use of cannabis increases the risk of using a hard drug such as heroin and cocaine later on, i.e. it claims that there is a causal relationship running from cannabis to hard drugs. By employing a number of different econometric approaches, empirical work has examined whether such a causal link exists. Some studies report evidence for a "gateway" effect of previous cannabis use (e.g. DeSimone, 1998, Fergusson et al., 2006, Bretteville-Jensen et al., 2008 and Melberg et al., forthcoming), while others (e.g. Pudney, 2003, van Ours, 2003, Beenstock and Rahav, 2002) find only weak or no support for such an effect. Despite the mixed and limited evidence for a gateway effect, the hypothesis has been influential in formulating a strict drug policy in many Western countries and opponents of decriminalizing cannabis often refer to the assumed causal relationship in their argumentation.

Proneness and Accessibility have been suggested as two alternative non-causal explanations for the observed correlation between cannabis and hard drug use and may lead to new policy implications. Proneness refers to personal factors that lead to a predisposition towards "problem behaviour" (Pudney, 2003). Such proneness to deviant behavior may lead some subjects to 
consume illicit drugs, and cannabis is just used prior to others as it is cheaper and more readily available. Excessive drug use is then viewed as one out of many potential undesirable behavioral responses, for example to unfavorable genetic endowments or traumatic childhood experiences. If people with this vulnerability have an increased risk for drug use, there may be no causal link between cannabis and hard drugs that explain the familiar initiation pattern (Morral et al., 2002).

Accessibility refers to a subject's economic, cultural and physical access to drugs and affects a subject's monetary and non-monetary costs of drug use, such as transaction costs and social stigma. Economic theory would predict a higher probability of drug uptake for subjects with better access (lower costs). Accessibility is likely to vary between youngsters. Some may have better knowledge of how and where to obtain drugs, what prices to pay etc. since, for example, drug use is more accepted in some youth cultures than others. If cannabis users are those subjects who tend to have lower costs of hard drug use this would contribute to the observed pattern of higher hard drug use in this group.

In this paper we investigate the role of proneness and accessibility in addition to that of previous cannabis intake on an individual's decision to initiate hard drug use. Detailed drug use data with information on both proneness and accessibility are rare. As a result little has been said about the effect of proneness and accessibility in recent gateway studies, which mainly rely on econometric techniques to isolate the gateway effect of previous cannabis use from that of unobserved proneness and accessibility. Exceptions include Pudney (2003) who uses prevalence indices (based on seizures, drug-related convictions and prevalence rates indicators) and Melberg et al. (forthcoming) who employ drug prices. For our analysis we exploit a unique survey of young Norwegians from 2006 that collected detailed data on drug use and initiation patterns, and also provides subject-level information on drug accessibility and proneness.

We take into account various possible channels for the gateway effect as a drug policy, aiming at reducing hard drug use, will depend not only on the role of accessibility and proneness factors versus a possible gateway effect, but also on the mechanisms that mediate a gateway effect of cannabis. A number of possible transmission channels have been suggested (see e.g. 
Pudney, 2003, Melberg et al., forthcoming): 1) the use of cannabis may create a psychological or physiological desire for stronger narcotic experiences; 2) personal experiences with cannabis may reduce the credibility in warnings against hard drugs; 3) having used one illegal drug may lower the threshold for trying other illegal drugs; 4) obtaining and using cannabis may induce contact with hard drugs users and dealers whom they would not otherwise have met. Channels 2 and 3 imply lower costs of hard drug use for cannabis user. This suggest a possible identification strategy for these channels as we can exploit the fact that a cannabis user would be more likely to move on to hard drugs than a non-users given the same accessibility. An epidemiological study by Wagner and Anthony (2002) has found that cannabis users have more opportunities to use other drugs and, given the same opportunity, face an increased risk of using other drugs.

We base our empirical analysis of the gateway, proneness and accessibility effects on a bivariate probit model of hard drug initiation and previous cannabis use that has features of an endogenous switching regression model. The modeling framework relaxes two common assumptions made in the previous empirical work: (1) constant gateway effect; and (2) same correlation pattern of the unobservables for all subjects. Our model is formulated within the Bayesian paradigm. We exploit information from a similar (but more restrictive) 2002 survey of young Norwegians to formulate our prior assumptions about the model parameters. We employ the Bayesian predictive approach to analyze the relative importance of the three effects on the probability of hard drug initiation. We estimate the marginal predictive probabilities of hard drug uptake under no and previous cannabis intake to quantify the effect of previous cannabis use and compute, for example, average marginal effects of accessibility, proneness and previous cannabis use. We also use the predictive approach to investigate various policy relevant scenarios.

The estimation of the model and the predictive analysis are based on Markov Chain Monte Carlo simulation methods, thus avoiding a solution via the optimization of the likelihood which can be challenging in such problems. Another advantage of the Bayesian predictive approach is that the predicted probabilities are based on the posterior distribution of the parameters rather than point estimates. Given the limited availability of strictly exogenous control variables in this type of problems a number of coefficients are estimated with a lower precision as reflected 
in the variance of the posterior distributions of the model parameters. In the predictive analysis under the Bayesian paradigm this imprecision is accounted for directly according the posterior distribution of the model parameters. Suitable Markov Chain Monte Carlo methods for the model fitting and predictive analysis are developed in the paper, building on work by Chib (2007) and Chib and Jacobi (2007).

Overall, a robust finding that emerges from our analysis is that proneness, accessibility and previous cannabis use contribute to the observed higher drug use pattern among cannabis users. The latter has the largest effect and is driven by various transmission channels.

\section{Data}

\subsection{The Survey}

In 2002 and 2006 the Norwegian institute for alcohol and drug research (SIRUS) sent postal questionnaires to a representative sample of young adults to collect data about the respondents' legal and illegal drug use. In this paper we employ the most recent of these surveys which includes data on ethnicity and for which we can construct price variables. The rich data set provides information on the frequency of current and life time drug use, as well as on the starting ages for the various drugs. In addition to personal characteristics, several variables related to proneness and accessibility to various drugs are available for each respondent. The survey was aimed at the population between the age of 21 and 30. This age group is particularly well suited for the analysis of illicit drug use behaviour, as these and other data show that most recreational drug users have started drug use by the ages of 21 and that frequency and extent of drug use is highest among young adults.

The response rate of the 2006 mail survey was 43 per cent, which is roughly in line with other studies of this type (see e.g. Reinarman et al., 2004). Only one reminder was sent to everyone. Our net sample consists of 4164 observations. Compared to another Norwegian data set that includes the same age group (for details see Horverak, 2006), the life-time prevalence rates for the different drugs are slightly higher in the present sample but within the same range (e.g. $32.5 \%$ vs $29.0 \%$ for cannabis). The lower rates in the other data set may be due to differences 
in data collection methods as it was based on face-to-face interviews for questions related to alcohol and a self-administered questionnaire for illegal drugs. A longitudinal study based on a representative sample of students in 1992 (mean age was 15 years) that were followed to 2005 also shows comparable prevalence rates for drug use (Pedersen, 2008).

It is well known, however, that various sub-groups (institutionalized people, intravenous/heavy drug users etc.) are likely to be under-represented in net samples from national surveys as these groups are harder to reach and may be less willing to fill out a questionnaire. Our findings may thus be more relevant for the group of recreational users of illicit and licit drugs than for the group of problematic drug users.

Figure 1 shows the initiation pattern of drug use in terms of the mean starting ages for alcohol and the main illegal drugs (cannabis, amphetamine, heroin, ecstasy and cocaine, respectively) in our sample. The graph reflects the commonly reported initiation pattern for drug use with

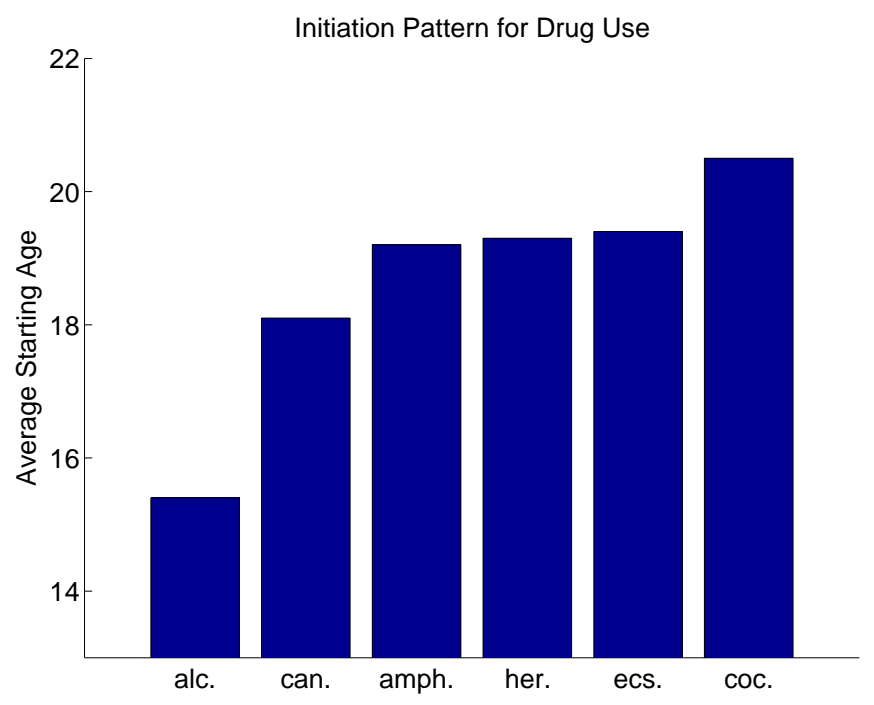

Figure 1: Starting ages for alcohol and various illegal drugs in the 2006 SIRUS survey.

cannabis being the illegal drug with the lowest starting age. The mean age of starting with alcohol is 15.4 which is well below the mean starting ages for illegal drugs: 18.1 for cannabis; 19.2 for amphetamine; 19.3 for heroin; 19.4 for ecstasy and 20.5 for cocaine. Since this paper is primarily concerned with a subject's involvements in the illegal drug market beyond cannabis use, we follow Melberg et al. (forthcoming) and merge the heroin, amphetamine, ecstasy and 
cocaine use variables into one hard drug use variable. All these drugs score higher than cannabis on "mean physical harm" and "acute harm" in a ranking made by a group of drug experts (Nutt et al., 2007). Also, the majority of the hard drug users in the survey (65\%) report to have used more than just one of these drugs. $80 \%$ or more of the amphetamine,cocaine, ecstasy and heroin users have use at least one other hard drug and $50 \%$ have used two or more other drugs (see Table 7 in the Appendix B).

The sampling procedure employed by SIRUS implies an over-sampling of people from the capital city, Oslo. This increases the overall prevalence of drug use as young people in Oslo use more drugs than people from comparable age groups outside the capital, but should not otherwise affect the sample. Norways drug use is typical of Europe except that Norway, together with Sweden and Finland, has considerably lower cannabis use relative to its affluence. According to national surveys of $15-34$ year olds, the life time cannabis use in Norway is $25.5 \%$ (Sweden 19.1\% and Finland 22.0\%)which is lower than in e.g. Denmark (49.5\%), U.K (42.3\%), France (43.6\%) and the Netherlands (32.3\%)(EMCDDA 2007). A recent report from the United Nations confirms that cannabis is even more prevalent in Oceania and North America than in Europe (UN 2007).

Good and reliable data on illicit drug use is difficult to obtain. General population surveys, like the present, are based on self-reported information and may suffer from false negatives (drug users claiming not to have used illegal substances or reporting a lower consumption level or frequency than the real one) and/or false positives (people exaggerating their actual drug use). For phenomena with low frequency, like heroin use, the false positives are considered a bigger problem than the false negatives (Skog 1992). We have no means to test for false negatives. However, the reported prevalence of the non-existing drug "relevin" gives an indication of false positives in the present sample. "Relevin" was listed as just another drug the respondents were asked about and with only 9 people in the sample ( 0.2 per cent) reporting to have ever used this particular drug, false positives does not seem to be a pervasive problem in our data. Since the data is based on a younger cohort it is reasonable to assume that recall bias is less of a problem compared to studies including people in their 40-60s. For most subjects in these older cohorts 
drug initiation would have occurred two or more decades before the time of the survey.

\subsection{Drug Use, Accessibility and Proneness in the Data}

Drug users were asked to report their frequency of illicit drug use (the categories were 0, 1-4, $5-10,11-25,26-50,50+)$. Life time prevalence of cannabis use in the current sample is 38.2 per cent. Many subjects report to have tried cannabis less than five times (see Table 13 in the appendix). It is questionable, however, whether using cannabis once or a few times only is sufficient to cause an effect on hard drug use. For our main empirical analysis we therefore assume that for cannabis to have an effect on further drug involvement a life time frequency of 5 or more times is required, and categorize "incidental" cannabis users (1-4) as non-users of cannabis. We relax this assumption in our sensitivity analysis. In the case of hard drug use, we define a subject as a user if any hard drug has been used at least once (including subjects from 1-4 category). Using a hard drug just once increases the risk of adverse health effect (heroin overdosing etc.). Given these definitions, the cannabis prevalence in the sample is $23.4 \%$ and the hard drug prevalence is $13.7 \%$. Among the 973 cannabis users, $48 \%(\mathrm{n}=469)$ have also used hard drugs, while $18 \%$ of the 571 hard drug users $(n=102)$ do not report any previous cannabis use.

Table 1 presents a summary of the sample means for relevant demographic, proneness and accessibility related variables by three different drug use states: no drug use; cannabis use only; hard drug use. We observe little variation in the age of subjects in the three groups: $29 \%$ of the sample respondents that have used no illegal drugs or only cannabis are 24 years or younger at the time of the interview compared to $27 \%$ of the hard drug users. The proportion of males, however, increases with drug involvement from $36 \%$ among the non-users to $52 \%$ among the hard drug users.

Individual proneness to drug use can be for example result of genetic factors such as a risk-seeking personality, association with "wrong" peers, neglect by parents. Fergusson et al. (2006) use covariates like conduct problems at age 14, school drop outs (leave school before the mandatory number of years) and alcohol and cigarette use at age 14 as proxies for individual proneness to drug use. Our data set provides several childhood-related variables that can be 


\begin{tabular}{ll|ccc}
\hline \multicolumn{1}{c}{ Variable } & No Drug Use \\
Description & [short] & $\begin{array}{c}\text { Cannabis (only) } \\
(\mathrm{n}=3,089)\end{array}$ & $\begin{array}{c}\text { Hard Drugs } \\
(\mathrm{n}=504)\end{array}$ \\
\hline $\begin{array}{l}\text { Demographics } \\
\text { Male }\end{array}$ & [male] & 0.36 & 0.43 & 0.52 \\
$\begin{array}{l}\text { Age between 21-24 years } \\
\text { Proneness }\end{array}$ & [age2124] & 0.29 & 0.29 & 0.27 \\
Childhood problems with Police & [police] & 0.007 & 0.004 & 0.06 \\
Childhood problems with Parents & [parents] & 0.06 & 0.10 & 0.20 \\
Childhood problems in School & [school] & 0.04 & 0.07 & 0.13 \\
Childhood problems with Friends & [friends] & 0.03 & 0.04 & 0.06 \\
Leave School after year 10 & [leaveschool] & 0.04 & 0.04 & 0.12 \\
Alcohol used before age 13 & [alcyoung] & 0.04 & 0.10 & 0.16 \\
Smoker & [smoke] & 0.45 & 0.86 & 0.84 \\
Accessibility & & & & 0.04 \\
Non-western cultural background & [nonwest] & 0.06 & 0.02 & 0.27 \\
Living in the Country & [country] & 0.42 & 0.24 & 0.55 \\
Obtain cannabis & [obtaincan] & 0.54 & 0.55 & 0.43 \\
Obtain hard drug & [obtainhard] & 0.42 & 0.43 & 0.25 \\
Cannabis prevalence & [prevcan] & 0.23 & 0.25 & 0.09 \\
Hard Drug prevalence & [prevhard] & 0.08 & 0.09 & 374 \\
\# of heroin seizures & [seizure] & 294 & 366 & 101 \\
Cannabis price & [canprice] & 101 & 100 & 545 \\
Amphetamine price & [amhprice] & 542 & 539 & 0.67 \\
Living in Oslo & [oslo] & 0.50 & 0.66 & \\
\hline
\end{tabular}

Table 1: Summary of key variables of 2006 sample of the SIRUS data by drug intake.

taken as proxies for proneness to drug use. For example, the respondents were asked "When thinking back to your childhood, would you say that you had any serious problems with parents, school, friends or police?" The term "childhood" is not specifically defined in the questionnaire. In accordance with the proneness hypothesis, Table 1 shows that these indicators have higher means among hard drug users than among the other two groups. Very few subjects in the non-user and cannabis-only categories report any problems with police (less than 1 per cent), compared to 6 per cent among the hard drug users. Problems with parents and school show an increasing trend when moving from non-users to hard drug users, with the latter group reporting three times the prevalence of non-users. Further, a larger proportion of hard drug users report problems with friends during childhood (6\%) than did cannabis-only and non-users of drugs. The large majority of young adults (86\%), however, did not claim any of the listed childhood problems and most of those who did only reported one type of problem (68\% of 578 individuals, see Table 9 in the Appendix B).

Table 1 further reveals that the group of illicit drug users has a much higher proportion of 
respondents that started with alcohol at a very young age $(<13$ years) and who dropped out of school after only 10 years (at the age of 15/16). Starting with alcohol before turning 13 or leaving school at an early age could be signs of a deviant personality, reflect adverse personal experiences or suggest a relatively high time preference rate, all of which could increase the probability of problematic drug use. Finally, given the well-known health risks associated with cigarette smoking we will include a dummy for smoking (set equal to 1 if ever smoked with some regularity) and use this as an additional proneness proxy.

In addition to individual proneness, we also consider a subject's accessibility (cultural, economic and/or physical) to drugs to be an important determinant of drug use. The survey provides information on various aspects of accessibility that are related to monetary and nonmonetary costs of cannabis and hard drug use. For example, the drug use culture among non-western immigrants may differ from that of native Norwegians. Table 1 shows that the highest proportion of non-western immigrants is found among the non-users. Respondents living in cities may have easier access (both physical and cultural) to drugs compared to subjects living in more remote rural areas of Norway. The distribution of the dummy variable "country" (equal to one if the respondent lives outside one of the three main cities in Norway) across the three groups of users in Table 1 is in line with this view as the proportion of urban people is higher among the two groups of illicit drug users. A further inspection of the data reveals that a larger proportion of respondents living in cities claim to be able to obtain illegal drugs within 3 days (72\% vs $57 \%$ ) and have been offered illegal drugs ( $78 \%$ vs $59 \%)$. The obtain variable also differs substantially by cannabis intake (see Table 10 in Appendix B). Employing these variables directly, however, may cause severe endogeneity issues. Instead we use the individual information to create location specific obtain variables for cannabis and hard drugs. The obtain variables in Table 1 are defined as the percentage of non-users that can obtain cannabis or hard drugs within 3 days in each of the 19 counties in Norway. As an additional indicator of cultural accessibility, we have included variables for the mean county prevalence of cannabis and hard drugs. Even though the "prevalence" and the "obtain" variables do not show much variation across the groups in Table 1, we find in our empirical analysis that they influence the cannabis 
and hard drug uptake (see Table 2). An important variable that directly relates to the economic accessibility is a person's income. Unfortunately, the survey only contains current income information for survey respondents, and no historic information on the personal, parents' or family income at the time of the drug initiation. Also, a quarter of the respondents are students at the time of the survey.

Accessibility related data from two supplementary data sources have also been included. The "seizure" variable is based on the mean number of amphetamine and heroin seizures by customs and police for each county. In our analysis we use this variable, adjusted for the population size, as a proxy for the geographical variation in hard drug supply. Table 1 shows that hard drug users live in areas with the highest number of seizures per 100,000 inhabitants. Finally, previous research on cannabis use indicates that drug prices are potentially important in explaining drug use as they are a direct measure of monetary costs of drug use (Van Ours and Williams, 2007). Given the illegality of drug markets, reliable and detailed price data are rare. We are fortunate to have data on cannabis and amphetamine prices that were collected through personal interviews with drug addicts. Since 1993 more than 4000 interviews have been conducted with people attending the main needle exchange service in Oslo (for more details see Bretteville-Jensen and Biorn, 2004). The prices for hard drugs have shown a falling trend throughout the period and the amphetamine price series is used as a proxy for all hard drug prices. Since we have crosssectional data, every person is assigned the cannabis and amphetamine price that prevailed when he/she statistically had the highest risk of taking up the different drugs (drug price and seizure variables are divided by 1000 in the estimations). We include a dummy for Oslo in our empirical analysis as the police reports that drug prices outside the capital are higher. However, this dummy would also capture differences in cannabis or hard drug culture in Oslo compared to other parts of Norway. 


\section{Empirical Analysis}

\subsection{Model Specification}

In this section we formulate a model to investigate the effect of prior cannabis use, accessibility and proneness factors on hard drug initiation in the Norwegian drug use data. In the empirical gateway literature a subject's hard drug intake has commonly been modeled as a function of a vector of (mostly demographic) variables $\mathbf{w}_{i}$ and an indicator $c_{i}$ for the subject's cannabis use prior to his or her hard drug initiation. If we let $h_{i}^{*}$ denote the underlying continuous latent hard drug initiation variable such a model for hard drug initiation can be written as

$$
h_{i}^{*}=\alpha^{0}+\mathbf{w}_{i}^{\prime} \boldsymbol{\alpha}+\alpha^{c} c_{i}+\varepsilon_{i}
$$

where the indicator for hard drug initiation $h_{i}$ is defined as $h_{i}=I\left[h_{i}^{*}>0\right]$. Assuming a normal link function, $\varepsilon_{i} \sim N(0,1)$, the specification implies a probit model for $h_{i}$. The coefficient $\alpha^{c}$ is commonly interpreted as the gateway effect of cannabis use on hard drug initiation. While it can be disputed under what conditions this coefficient can be interpreted as a causal effect, we will follow the literature and refer to it here as the constant/direct gateway effect. Since it is not possible to rule out the presence of unobserved factors that affect both a subject's probability to use cannabis as well as harder drugs, the recent literature has treated $c_{i}$ as an endogenous regressor.

For our analysis of accessibility, proneness and previous cannabis use on hard drug initiation, we propose a modeling framework in terms of more flexible bivariate probit models with features of an endogenous switching regression model, allowing for example for different gateway transmission channels. We specify bivariate probit models for the hard drug initiation $h_{j i}=l$ and previous cannabis use $c_{i}=j$ of the $i$ th subject, where $l, j=0,1$. The notation $h_{j i}$ emphasizes that the model for hard drug initiation will depend on the subject's previous cannabis use, indicated by the subscript $j$. We set the indicator for previous cannabis use equal to one if the age of cannabis initiation is lower than the age of onset for any of the hard drugs. A small number of subjects in our sample $(n=101)$ report the same starting age for both cannabis and a hard drug. In those cases we have assumed the common initiation pattern with cannabis preceding 
hard drugs initiation. For subjects that use cannabis, but initiated cannabis use after hard drug use, $c_{i}$ is set to zero. This recursive specification follows the approaches in the gateway literature adopted by Pudney (2003), van Ours (2003) and Bretteville-Jensen et al. (2008). As pointed out in the latter, bi- and multivariate probit models can be used to estimate causal effects of an endogenous binary regressor in a recursive model, which follows for example Maddala (1983), Greene (2002) and Contoyannis and Jones (2004).

We specify the marginal models for binary hard drug initiation and previous cannabis use in terms of the latent variables as

$$
\begin{aligned}
h_{j i}^{*} & =\alpha^{0}+\mathbf{w}_{a h, i}^{\prime} \boldsymbol{\beta}_{j}+c_{i} \alpha^{c}+\mathbf{w}_{p, i}^{\prime} \boldsymbol{\alpha}^{p}+\mathbf{w}_{d, i}^{\prime} \boldsymbol{\alpha}^{d}+\varepsilon_{j i}, c_{i}=j \\
c_{i}^{*} & =\gamma^{0}+\mathbf{w}_{a c, i}^{\prime} \gamma^{a}+\mathbf{w}_{p, i}^{\prime} \gamma^{p}+\mathbf{w}_{d, i}^{\prime} \gamma^{d}+u_{i}
\end{aligned}
$$

where $h_{j i}=I\left[h_{j i}^{*}>0\right]$ and $c_{i}=I\left[c_{i}^{*}>0\right]$. The error terms are jointly normally distributed, as a function of previous cannabis use $c_{i}=j$, as

$$
\varepsilon_{j i}, u_{i} \sim N\left(0, \boldsymbol{\Omega}_{j}=\left(\begin{array}{cc}
1 & \rho_{j} \\
\rho_{j} & 1
\end{array}\right)\right) .
$$

The covariate vectors for accessibility, proneness and demographic factors are defined in terms of the variables described in Table 1 as

$$
\begin{aligned}
\mathbf{w}_{a h, i} & =[\text { country, oslo, obtainhard, seizure, prevhard,nonwest,amphprice }] \\
\mathbf{w}_{p, i} & =[\text { police}, \text { parents, school, friends, leaveschool, alcyoung, smoke }] \\
\mathbf{w}_{d, i} & =[\text { male, age } 2124] \\
\mathbf{w}_{a c, i} & =[\text { country, oslo, obtaincan, prevcan, nonwest, canprice }] .
\end{aligned}
$$

The model extends the standard framework to consider the role of proneness and accessibility factors on drug initiation and relaxes two restrictive assumptions. First the model relaxes the assumption of a constant/homogeneous (gateway) mean effect of previous cannabis use on hard drug initiation. Hard drug initiation (3.2) is now modeled as a function of accessibility factors $\left(\mathbf{w}_{a h, i}\right)$, proneness factors $\left(\mathbf{w}_{p, i}\right)$ and previous cannabis use. Previous cannabis use affects the mean of the hard drug initiation model through (i) a constant effect modeled $\left(\alpha^{c} c_{i}\right)$ and (ii) through interaction with the vector of accessibility factors $\left(\mathbf{w}_{a h, i}^{\prime} \boldsymbol{\beta}_{j}\right)$. Our model thus implies 
that the overall effect of prior cannabis use on hard drug initiation is captured by a constant effect and heterogeneous effect which are motivated by the different transmission channels. The latter heterogeneous effect is identified through different coefficient vectors on the accessibility variables, $\boldsymbol{\beta}_{0}$ for subjects with no previous cannabis use and $\boldsymbol{\beta}_{1}$ for subjects with previous cannabis use. The constant/homogeneous and heterogenous effects of previous cannabis use on hard drug initiation can be identified since we have kept the assumption of a common intercept $\alpha^{0}$. We refer to the composite effect of previous cannabis use as the extended gateway effect in the remainder of the paper and to this model as the "Extended Gateway model".

There are several reasons why one may wish to relax the assumption of modeling the effect of previous cannabis use through only a constant "gateway" effect and proceed with our proposed specification. First, as the discussion in the introduction highlights, there are at least four possible transmission channels by which cannabis use may affect a subject's likelihood of hard drug initiation: 1) creates psychological or physiological desire for stronger narcotic experiences; 2) reduces credibility in warnings against hard drugs; 3) lowers threshold for trying harder drugs; 4) leads to better contact and knowledge about the hard drug market. Second, we are interested in exploring the role of proneness and accessibility factors on hard drug initiation. If cannabis use affects hard drug initiation through channels (2) and (3), this would imply that subjects with previous cannabis use are more likely to initiate hard drug use given the same cost of hard drug initiation as measured by cultural and physical accessibility factors. We have therefore modeled the effect of accessibility as a function of a subject's previous cannabis use. In the presence of channels (2) and (3) we would expect to see different posterior distributions for the coefficients in $\boldsymbol{\beta}_{0}$ and $\boldsymbol{\beta}_{1}$. We still include a constant effect of previous cannabis use measured by $\alpha^{c}$ to capture channels (1) and (4). A posterior distribution of $\alpha^{c}$ with a positive mean would be a support for either one or both of these channels.

Second, our modeling framework relaxes the assumption of the same unobserved correlation patterns between cannabis and hard drug use for all subjects (for example cannabis users and non-users). The raw data shows that cannabis users and non-users differ significantly in terms of their observed characteristics and thus, very likely also in terms of their unobserved factors 
that affect cannabis and hard drug initiation. For example, we cannot rule out that cannabis users differ in terms of their unobserved proneness characteristics or socioeconomic background from the non-cannabis users in the population. These factors may affect cannabis and hard drug uptake, but not necessarily in the same way, which can be captured by different correlation pattern for cannabis users and non-users.

Another interesting feature of our modeling approach is that we exploit the information on accessibility and proneness factors in the modeling of cannabis initiation (3.3). By modeling previous cannabis intake in such a way we obtain a benchmark for the interpretation of the coefficient estimates from the hard drug model and also a more differentiated picture of hard drug versus cannabis use.

To benchmark our coefficient estimates for the constant gateway effect, we also consider a simpler specification of the hard drug model similar to those in previous work and only include a constant gateway effect of cannabis use ("Restricted Gateway Model"). In our model specification (3.1) this would be reflected by setting $\boldsymbol{\beta}_{0}=\boldsymbol{\beta}_{1}$. Note that while it would be theoretically possible to fit a fully flexible hard drug model where all coefficient vectors depend on previous cannabis intake, this would not allow us to identify the coefficient on the constant gateway effect as the effect would be subsumed in the intercept. In addition, the very low prevalence of some proneness variables in the group of non-drug users would make it very difficult to identify $\boldsymbol{\alpha}_{j}^{p}$.

Finally, to express our joint model for hard drug initiation and previous cannabis use more compactly and to aid our formulation of prior parameters, we define the parameter vectors $\boldsymbol{\alpha}=\left\{\alpha^{0}, \boldsymbol{\alpha}^{c}, \boldsymbol{\alpha}^{p}, \boldsymbol{\alpha}^{d}\right\}, \boldsymbol{\gamma}=\left\{\gamma^{0}, \boldsymbol{\gamma}^{a}, \boldsymbol{\gamma}^{p}, \boldsymbol{\gamma}^{d}\right\}$ and the covariate vectors $\mathbf{w}_{1, h i}=\left[\mathbf{w}_{a h, i}\right], \mathbf{w}_{2, h i}=$ $\left[\right.$ const, $\left.c_{i}, \mathbf{w}_{p, i}, \mathbf{w}_{d, i}\right]$ and $\mathbf{w}_{c i}=\left[\right.$ const $\left., \mathbf{w}_{a c, i}, \mathbf{w}_{p, i}, \mathbf{w}_{d, i}\right]$. We can now write the joint model for subject's latent hard drug initiation and previous cannabis use with $c_{i}=j$ in terms of the bivariate normal distribution

$$
\mathcal{N}_{2}\left(h_{j i}^{*}, c_{i}^{*} \mid \mathbf{W}_{j i}^{\prime} \boldsymbol{\delta}, \boldsymbol{\Omega}_{j}\right), j=0,1
$$

where

$$
\mathbf{W}_{j i}=\left(\begin{array}{cccc}
\mathbf{w}_{1, h i}^{\prime} \times(1-j) & \mathbf{w}_{1, h i}^{\prime} \times(j) & \mathbf{w}_{2, h i}^{\prime} & 0 \\
0 & 0 & 0 & \mathbf{w}_{c i}^{\prime}
\end{array}\right)
$$


and $\boldsymbol{\delta}=\left(\boldsymbol{\beta}_{0}, \boldsymbol{\beta}_{1}, \boldsymbol{\alpha}, \gamma\right)$.

\subsection{Prior-Posterior Analysis}

In the Bayesian estimation framework all information about the vector of model parameters, $\boldsymbol{\theta}=\left(\boldsymbol{\beta}_{0}, \boldsymbol{\beta}_{1}, \boldsymbol{\alpha}, \boldsymbol{\gamma}, \rho_{0}, \rho_{1}\right)$, is summarized in the posterior distribution of the model parameters conditional on the observed data $\pi(\boldsymbol{\theta} \mid \mathbf{h}, \mathbf{c}, \mathbf{W})$. The posterior distribution combines the information from the data, via the likelihood

$$
p(\mathbf{h}, \mathbf{c} \mid \mathbf{W}, \boldsymbol{\theta})=\prod_{i=1}^{n} \prod_{j=0}^{1} \operatorname{Pr}\left(h_{j i}=l, c_{i}=j \mid \mathbf{W}_{i}, \boldsymbol{\theta}\right)
$$

and prior knowledge about the parameters expressed in the prior distribution $\pi(\boldsymbol{\theta})$. From the modeling assumptions in the previous section it follows directly that the likelihood contribution of the $i$ th subject can be written in terms of the bivariate normal distributions of the latent drug variables $\left(h_{j i}^{*}, c_{i}^{*}\right)$ as

$$
\operatorname{Pr}\left(h_{j i}=l, c_{i}=j \mid \mathbf{W}_{i}, \boldsymbol{\theta}\right)=\int_{A_{l}} \int_{A_{j}} \mathcal{N}_{2}\left(h_{j i}^{*}, c_{i}^{*} \mid \mathbf{W}_{j i}^{\prime} \boldsymbol{\delta}, \boldsymbol{\Omega}_{j}\right) d c_{i}^{*} d h_{j i}^{*}
$$

where $l=0,1$, and the integration regions are $\{-\infty, 0\}$ for $l, j=0$ and $\{0,+\infty\}$ for $l, j=1$.

The prior-posterior analysis provides us with a tool to incorporate prior knowledge about the model parameters via the prior distributions of the parameters, $\pi(\boldsymbol{\theta})$. To formulate our prior assumptions we first follow standard practice and specify normal prior distributions for the slope parameters and the correlation coefficient. For the correlation coefficient the normal prior is restricted to the region $R=\left\{-1<\rho_{j}<1\right\}$ to ensure the positive definiteness of the correlation matrix $\boldsymbol{\Omega}_{j}$. Assuming that the parameters are a priori independent, the prior distribution of the coefficient vector, $\boldsymbol{\theta}$ is given by

$$
\pi(\boldsymbol{\theta})=\mathcal{N}_{m}\left(\boldsymbol{\alpha} \mid \boldsymbol{a}_{0}, \mathbf{A}_{0}\right) \mathcal{N}_{q}\left(\gamma \mid \boldsymbol{g}_{0}, \mathbf{G}_{0}\right) \prod_{j=0}^{1} \mathcal{N}_{p}\left(\boldsymbol{\beta}_{j} \mid \boldsymbol{b}_{j 0}, \mathbf{B}_{j 0}\right) \mathcal{N}\left(\rho_{j} \mid r_{j o}, R_{j 0}\right) \times R .
$$

Since the specific problem and data has not been previously analyzed in the literature we use information from the 2002 drug survey of SIRUS to help specify the parameters in the prior distributions (see Appendix A for details).

The posterior distribution of the parameters is proportional to the product of the likelihood (3.5) and the prior (3.7). Given the structure of the likelihood, it is not available in closed 
form and we develop an estimation algorithm based on Markov Chain Monte Carlo methods to generate draws from the posterior distribution. We employ the approach from Albert and Chib (1993) to deal with the binary variables by including the latent drug use variables into the parameter space. The details of the algorithm are given in the Appendix A. Of particular interest for our analysis are inferences based on the marginal posterior distribution of each parameter. Fortunately, by construction of the MCMC algorithm, the draws for each parameters come from the marginal posterior distribution of that particular parameter, which can be used to summarize the corresponding marginal posterior distributions of the model parameters. Usually this is done in terms of the means and standard deviations of the marginal posterior distribution and/or directly in graphical form.

\subsection{Predictive Analysis}

While the inferences about coefficients from the prior-posterior analysis will provide us with some information about the effects of the accessibility and proneness related variables and previous cannabis use on hard drug initiation, the coefficients of the probit model are not easily interpreted. Further, the coefficient estimates will not allow us to assess the overall impact of the observed accessibility and proneness related factors on a subject's probability of hard drug uptake compared to that of previous cannabis use. To address this issues we employ a predictive analysis of the probability of hard drug uptake.

The predictive approach enables us to draw inferences about the hard drug initiation of a random future observation from the population (denoted with $n+1$ ). It will be based on our observed sample data $(\mathbf{c}, \mathbf{h}, \mathbf{W})$ and the inferences about the model parameters summarized in the posterior distribution of the model parameters (from the model fitting). In this paper we are particularly interested in the probabilities that a random subject from the population will initiate hard drug uptake under no and previous cannabis use. We then explore how these probabilities are affected by accessibility and proneness related factors. We also explore the constant/direct effect of prior cannabis use on the probability of hard drug initiation.

The marginal predictive probabilities of hard drug use $\operatorname{Pr}\left(h_{j, n+1}=1 \mid \mathbf{c}, \mathbf{h}, \mathbf{W}\right), j=0,1$ can be computed based on the marginal predictive distribution of the latent hard drug intake for a 
subject as

$$
\operatorname{Pr}\left(h_{j, n+1}=1 \mid \mathbf{c}, \mathbf{h}, \mathbf{W}\right)=\operatorname{Pr}\left(h_{j, n+1}^{*}>0 \mid \mathbf{c}, \mathbf{h}, \mathbf{W}\right)=\int_{0}^{\infty} p\left(h_{j, n+1}^{*} \mid \mathbf{c}, \mathbf{h}, \mathbf{W}\right) d h_{j, n+1}^{*}
$$

The marginal predictive distribution is not of a known analytical form. We can estimate the integral from the marginal distribution of hard drug intake conditional on the model parameters $p\left(h_{j, n+1}^{*} \mid \mathbf{h}, \mathbf{c}, \mathbf{W}, \mathbf{w}_{h, n+1}, \boldsymbol{\beta}_{j}, \boldsymbol{\alpha}\right)$, which takes the form of a Normal density, by numerically integrating over the model parameters using the posterior distribution of the model parameters from the model fitting and over the covariates using the empirical distribution from the sample. As described in more detail in Appendix A we can extend the algorithm for the model estimation to generate draws from the predictive distributions that can be summarized in various ways.

To see how we use the predictive approach to assess the effect of accessibility, proneness and the direct gateway effect on the predictive probability, we express the marginal predictive distribution $p\left(h_{j, n+1}^{*} \mid \mathbf{h}, \mathbf{c}, \mathbf{W}\right)$ as

$$
\int p\left(h_{j, n+1}^{*} \mid \mathbf{h}, \mathbf{c}, \mathbf{W}, \mathbf{w}_{h, n+1}, \boldsymbol{\beta}_{j}, \boldsymbol{\alpha}\right) \pi\left(\boldsymbol{\beta}_{j}, \boldsymbol{\alpha} \mid \mathbf{h}, \mathbf{c}, \mathbf{W}\right) p\left(\mathbf{w}_{h, n+1} \mid \mathbf{h}, \mathbf{c}, \mathbf{W}\right) d \boldsymbol{\beta}_{j} d \boldsymbol{\alpha} d \mathbf{w}_{h, n+1}
$$

where the first expression is the conditional predictive distribution, $\pi\left(\boldsymbol{\beta}_{j}, \boldsymbol{\alpha} \mid \mathbf{h}, \mathbf{c}, \mathbf{W}\right)$ is the posterior distribution of parameters in the marginal probit models for hard drug initiation and $p\left(\mathbf{w}_{h, n+1} \mid \mathbf{h}, \mathbf{c}, \mathbf{W}\right)$ the empirical distribution of the covariates from the hard drug model. The conditional predictive distribution is just the likelihood of the future observation $n+1$, given by the marginal model for latent hard drug intake $h_{j, n+1}^{*}$ by expression (3.2). From the model assumptions in Section 3.2 it follows that it is given by a normal distribution $\mathcal{N}\left(h_{j, n+1}^{*} \mid \boldsymbol{\mu}_{j, n+1}, 1\right)$ where the mean is a function of cultural and physical accessibility factors, prices, proneness and demographic factors:

$$
\boldsymbol{\mu}_{j, n+1}=\alpha^{0}+\mathbf{w}_{a h, n+1}^{\prime} \boldsymbol{\beta}_{j}^{a}+\alpha^{c} c_{n+1}+\mathbf{w}_{p, n+1}^{\prime} \boldsymbol{\alpha}^{p}+\mathbf{w}_{d, n+1}^{\prime} \boldsymbol{\alpha}^{d}
$$

where $j=1$ refers to $c_{n+1}=1$ and $j=0$ to $c_{n+1}=0$. To assess how a subject's probability of hard drug uptake is influenced by the three factors we also compute the predictive probabilities setting $\boldsymbol{\beta}_{j}^{a}=0, \alpha^{c}=0$ or $\boldsymbol{\alpha}^{p}=0$ (see Table 3). Comparing the obtained predictive probabilities without the effects of observed accessibility with the total predictive probability allows us to 
evaluate the average marginal effect of accessibility in predicting a subject's hard drug uptake. For example, estimating the partial predictive probability for $j=0,1$ under $\boldsymbol{\alpha}^{p}=0$ the difference to the predictive probability can be interpreted as the average marginal effects of proneness on hard drug initiation under no and previous cannabis use. Since this calculation can only be based on observed accessibility, these results should be interpreted with care and understood more as a lower limit of the role that accessibility factors play in a subject's hard drug uptake.

\section{Estimation Results}

\subsection{Results from Prior-Posterior Analysis}

In this section we discuss the results from the prior-posterior and predictive analysis of the Norwegian drug use data based on the extended gateway model that we described in the previous section. Table 2 provides a summary of the estimation results for the extended gateway model in terms of the means and standard deviations of the posterior distributions of the parameters. Our results are based on 10,000 iterations of the MCMC algorithm (described in detail in the Appendix A) after a burn-in phase of 1,000 iterations. For comparison, we also provide selected results from the fitting of a more restricted model with only a constant effect of previous cannabis use on hard drug uptake (restricted gateway model).

A comparison of the means of the posterior distributions of the coefficients on the accessibility factors in the hard drug model (first block in columns 2 and 3) indicates that accessibility affects hard drug initiation and that the effect varies by previous cannabis use. For example, we see that living in the country increases the likelihood of a cannabis user to take up hard drugs, as does living in an area with high supply (seizure), high prevalence and being of non-western background. The latter has a negative effect for non-cannabis users as does living outside Oslo. One possible interpretation is that for people in the countryside and for non-Westerns, drug use seems to be closer to an all or nothing decision; if you use cannabis, you are likely to go on to use hard drugs. In contrast, for native Norwegians in Oslo, it appears that cannabis use is more normative, which makes it is more likely for someone to use cannabis but not use hard drugs. The effects of the remaining accessibility variables are small and estimated with a lower precision 


\begin{tabular}{|c|c|c|c|c|}
\hline & \multicolumn{3}{|c|}{ Extended Gateway Model } & \multirow{3}{*}{$\begin{array}{c}\text { Restricted Gateway Model } \\
\text { Hard uptake }\end{array}$} \\
\hline & \multicolumn{2}{|c|}{ Hard uptake } & \multirow[t]{2}{*}{ Can uptake } & \\
\hline & no can & can & & \\
\hline Country & $0.12(0.19)$ & $0.37(0.18)$ & $-0.27(0.10)$ & $0.17(0.14)$ \\
\hline Oslo & $0.46(0.28)$ & $-1.11(0.46)$ & $-0.03(0.11)$ & $-0.06(0.25)$ \\
\hline Obtain & $0.48(0.99)$ & $-0.02(1.06)$ & $0.15(0.71)$ & $0.05(0.75)$ \\
\hline Seizure & $0.11(0.67)$ & $3.21(1.11)$ & - & $0.97(0.61)$ \\
\hline Prevalence & $0.20(1.31)$ & $2.46(1.97)$ & $2.26(0.91)$ & $1.47(1.23)$ \\
\hline Non-Western & $-0.31(0.19)$ & $0.48(0.29)$ & $-0.54(0.13)$ & $-0.08(0.15)$ \\
\hline Amphetamine Price & $0.47(0.41)$ & $0.04(0.45)$ & - & $0.23(0.35)$ \\
\hline Cannabis Price & - & - & $-0.62(1.38)$ & - \\
\hline Cannabis Use & 1.20 & $0.54)$ & - & $1.10(0.41)$ \\
\hline Police prob. & 0.90 & $0.22)$ & $0.62(0.19)$ & $0.89(0.21)$ \\
\hline Parents prob. & 0.45 & $0.11)$ & $0.42(0.08)$ & $0.46(0.10)$ \\
\hline School prob. & -0.02 & $0.13)$ & $0.25(0.11)$ & $0.01(0.13)$ \\
\hline Friends prob. & -0.04 & $0.16)$ & $-0.09(0.13)$ & $-0.02(0.15)$ \\
\hline Leave School early & 0.52 & $0.12)$ & $0.19(0.11)$ & $0.50(0.12)$ \\
\hline Alcohol young & 0.28 & $0.11)$ & $0.41(0.09)$ & $0.31(0.11)$ \\
\hline Smoker & 0.42 & $0.15)$ & $1.17(0.06)$ & $0.41(0.16)$ \\
\hline Male & 0.28 & $0.07)$ & $0.43(0.05)$ & $0.28(0.08)$ \\
\hline Age2124 & -0.01 & $0.10)$ & $0.03(0.06)$ & $-0.02(0.09)$ \\
\hline Intercept & -2.81 & $0.48)$ & $-2.27(0.39)$ & $-2.65(0.37)$ \\
\hline Correlation & $0.34(0.23)$ & $0.06(0.20)$ & & $0.26(0.14) \quad 0.15(0.21)$ \\
\hline
\end{tabular}

Table 2: Posterior means and standard deviations (parentheses) for parameters in the cannabis and hard drug equation from the prior posterior analysis of the extended gateway model and the hard drug equation from the restricted gateway model.

for non-cannabis users as indicated by the high posterior standard deviation. The low precision is not surprising as there is only a small number of hard drug users without previous cannabis use in the data $(\mathrm{n}=102)$. Further, our estimates show almost no effect of amphetamine price on the probability of hard drug uptake of cannabis users (posterior mean of 0.04 and standard deviation of 0.45 ), while the effect is positive for non-users (posterior mean of 0.47 ) but also estimated with a low precision (posterior standard deviation of 0.41 ).

For previous cannabis use (column 4) the coefficient on cannabis price is negative, but also imprecisely estimated. This suggests that prices (as measured here) are not of great importance for drug initiation. However, we cannot rule out that this is, at least partly, due to the poor data situation (only data for Oslo is available) and to how the price variable is constructed. For example, van Ours and Williams (2007) find a negative effect of cannabis price on cannabis initiation. The remaining coefficient estimates for the accessibility variables in the cannabis 
model suggest that accessibility factors also matter for cannabis intake. Cannabis prevalence and obtainability have a positive effect on cannabis use while being of non-western cultural background and living in the country has a negative effect. We find no effect for living in Oslo. For hard drug initiation we observe a positive coefficient for the Oslo variable under no previous cannabis use and a negative coefficient under previous cannabis use. The latter might suggest that a cultural acceptance of cannabis use in Oslo (larger prevalence of cannabis use in Oslo in the raw data) does not translate into a cultural acceptance for hard drug use.

We now turn to the estimates of the constant/direct effect of previous cannabis use and proneness factors. Table 2 shows a large positive coefficient for previous cannabis use on the probability of hard drug initiation. We also find positive coefficients for childhood problems with police and parents, leaving school early, early alcohol use and smoking. These factors also increase the likelihood of cannabis use. In contrast, problems in school and with friends do not seem to have an effect on hard drug initiation. We find similar results for the coefficients on previous cannabis use and the proneness variables in the hard drug model in our estimation of the restricted model that allows only for a constant effect of previous cannabis use (see last column of Table 2). However, this is not the case for the coefficients on the accessibility and price variables. We see that the coefficients on Oslo, Non-Western and the price variables are now close to zero. Except for living in the country, which has a positive effect, the coefficients on the remaining variables are estimated with a low precision.

We have set up our model to allow for different correlation patterns between the unobservables in the models for hard drug initiation and previous cannabis use in the sub-populations of drug users and non-users. We do observe differences in the estimated correlation patterns for the two groups. The posterior mean and standard deviation for the correlation coefficients $\left(\rho_{j}\right)$ for our extended gateway model and also the restricted model are shown in the last row in Table 2. We observe positive posterior means for $\rho_{0}$ and $\rho_{1}$ in both models. In the extended gateway model the posterior mean for non-users of cannabis exceeds that of cannabis users by more than five times (less than two times in the restricted model) and the latter is very small. Previous work that has used a more restrictive model formulation for hard drug use and the correlation 
structure has found a positive correlation for the overall population (Bretteville-Jensen et al., 2008), and under such restrictions we too estimate a positive correlation for both groups.

For a more detailed summary of the accessibility related coefficients in the hard drug model we take a closer look at the posterior distributions of the accessibility coefficients from the extended gateway model. In Figure 2 we provide plots of the posterior distributions of the coefficients for the accessibility variables in vectors $\boldsymbol{\beta}_{0}$ and $\boldsymbol{\beta}_{1}$. The solid line refers to no previous cannabis use and the dashed line to previous cannabis use. Except for the case of the obtain variable (Plot c), the two lines have different regions of support (some overlapping). These graphs thus provide further evidence for a heterogeneous gateway effect (in addition to

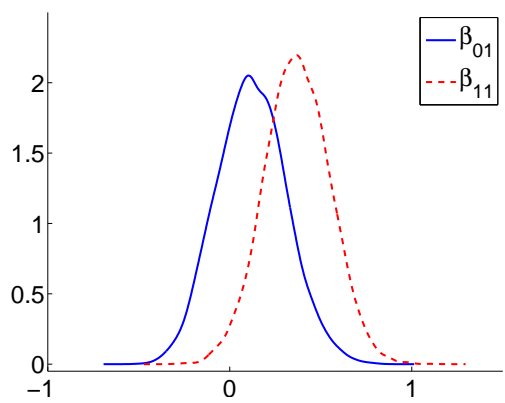

(a) Country

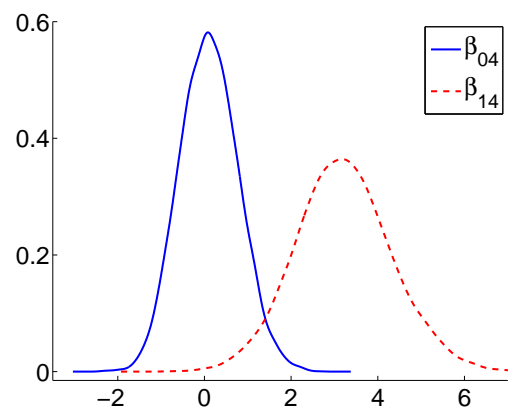

(d) Seizure

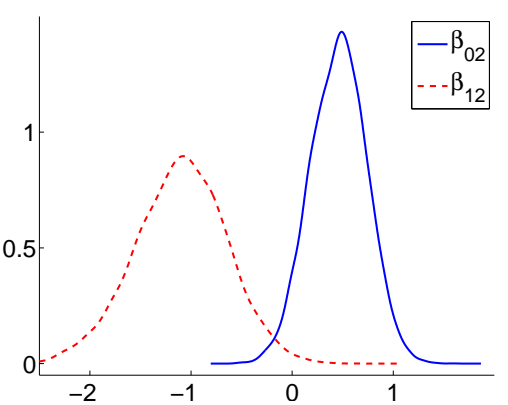

(b) Oslo

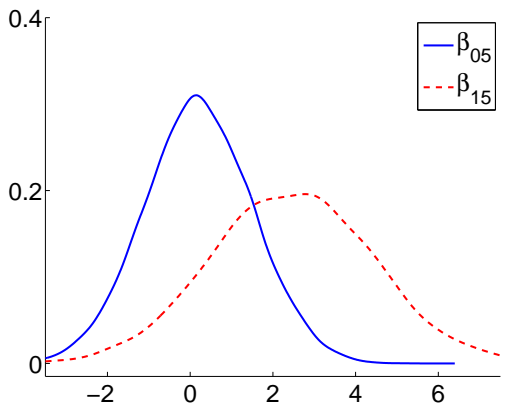

(e) Prevalence

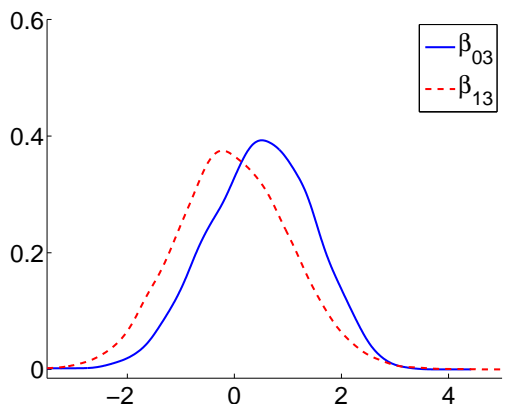

(c) Obtain

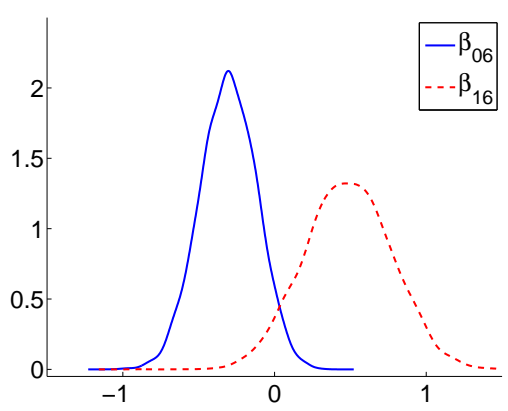

(f) Non-western

Figure 2: Plots of the posterior distributions of the coefficients for Oslo, prevalence and non-western under no cannabis use (solid line) and cannabis use (dashed line).

a constant gateway effect) as it shows that the impact of most accessibility variables on hard drug uptake depends on previous cannabis use. We therefore proceed with our further analysis, including the predictive analysis and policy analysis in the following sections, under the extended gateway model. In the next section we perform a sensitivity analysis of the extended gateway model and consider a range of alternative model specifications, e.g. alternative definitions for 
the dependent variables and alternative vectors of covariates.

Note that our estimates for the accessibility variables in the two hard drug models, presented in Table 2 and Figure 2, are interesting with regard to the possible transmission mechanisms of cannabis use. In particular, the fact that physical accessibility, measured by the seizure and prevalence variables, has a large positive effect under previous cannabis use compared to almost no effect under no previous cannabis use may suggest that having used cannabis lowers the threshold and the perceived risk of using hard drugs (transmission channel 2 and 3). Further, we find that previous cannabis use has a strong direct effect on hard drug uptake. This propose that cannabis use may create a desire for stronger drugs and/or yield better knowledge of the drug market (transmission channels 1 and 4). We further explore these issues in our sensitivity analysis in Section 6.

\subsection{Results from Predictive Analysis}

We employ the predictive analysis to explore the importance of accessibility and proneness related factors, in addition to that of previous cannabis intake, on a subject's probability of hard drug uptake. We first investigate the effects for a random (average) subject from the population. As described in Section 3.3 we focus on the marginal predictive probability of hard drug uptake under no and previous cannabis use, in particular the means of the predictive distributions $E\left[\operatorname{Pr}\left(h_{0, n+1}=1 \mid \mathbf{c}, \mathbf{h}, \mathbf{W}\right)\right]$ and $E\left[\operatorname{Pr}\left(h_{1, n+1}=1 \mid \mathbf{c}, \mathbf{h}, \mathbf{W}\right)\right]$. From the first row in Table 3 we see that the subject's mean probability to take up hard drugs is $7.7 \%$ under no previous cannabis use and $36.3 \%$ under previous cannabis use. These two probabilities refer to the two counterfactual scenarios if we were to observe the same random subject from the population under no and previous cannabis use. The estimates are based on the marginal model of hard drug uptake and are corrected for unobserved confounding (selection on unobservables). If we assume that we have successfully accounted for all unobserved confounders through our flexible joint model, the difference of 28.6 percentage points in the mean predictive probabilities can be interpreted as the average causal effect of cannabis use on hard drug uptake.

Table 3 also reports the estimates for the marginal predictive probabilities without the effect of accessibility, price and proneness factors as well as the direct "gateway" effect (under previous 


\begin{tabular}{lrrrr}
\hline & $\begin{array}{c}\text { Mean Predictive Probabilities for Extended Gateway Model } \\
\text { informed prior } \\
\text { no cannabis }\end{array}$ & cannabis & no cannabis & cannabis \\
\hline $\begin{array}{l}\text { Total } \\
\text { Partial }\end{array}$ & $0.077(.008, .298)$ & $0.363(.068, .771)$ & $0.081(.009, .318)$ & $0.351(.068, .762)$ \\
w/o Access* & $0.033(.001, .198)$ & $0.166(.011, .553)$ & $0.038(.001, .217)$ & $0.137(.007, .496)$ \\
w/o Prices & $0.053(.003, .248)$ & $0.359(.062, .789)$ & $0.061(.004, .280)$ & $0.348(.055, .748)$ \\
w/o Gateway & - & $0.092(.002, .419)$ & - & $0.129(.003, .509)$ \\
w/o Proneness & $0.053(.003, .248)$ & $0.258(.056, .557)$ & $0.061(.004, .280)$ & $0.245(.049, .549)$ \\
\hline
\end{tabular}

Table 3: Mean of the total and partial marginal predictive probabilities of hard drug uptake based on the extended gateway model under two different prior assumptions. 5\% and 95\% quantiles of the predictive distributions are given in parentheses. The informed prior uses information from the 2002 survey. Under the default prior the prior distributions for all parameters are centered at zero. *Access includes all access related variables from main specification other than prices.

cannabis use only). As discussed in Section 3.3, these "partial" probabilities are obtained by setting the corresponding coefficients to zero. From these we can then compute the average marginal effects. For example, the effect of the accessibility factors for non-users is 4.4 percentage points $(7.7 \%-3.3 \%)$. Under previous cannabis use, the average marginal effect of accessibility factors is considerably higher with 19.7 percentage points due to the extended gateway effect. The largest average marginal effect is that of the constant gateway effect with 27.1 percentage points. Without the constant gateway effect, the probability of hard drug initiation under cannabis use reduces to $9.2 \%$ and comes close to that under no previous use of $7.7 \%$. As expected from the estimates of the price coefficient, the effect of prices is the smallest.

We also report the predictive results for the analysis of the extended gateway model under alternative prior assumptions (default prior) that are not based on the 2002 data in Table 3. The estimates of the predictive probabilities as well as those of the model parameters (see Table 12 in Appendix B) are comparable to those from the analysis under the informed priors. We do observe a smaller decrease in the probability due to the direct gateway effect as result of the smaller mean of the posterior distribution for the constant effect of previous cannabis use (as shown in Table 2). Overall the estimates in Table 3 suggest that accessibility and proneness also play an important role in subject's hard drug initiation. Both proneness and accessibility have a larger effect on the probability of hard drug uptake for cannabis user, which contributes to the observed pattern of higher hard drug use among cannabis users. Since we can only assess 
the effects based on the available limited data on proneness and accessibility, it is very likely that our estimates should be interpreted as a lower bound of the effects, e.g. the intercept terms may include the effects of unobserved proneness and accessibility factors. We also note that especially the effect of accessibility related variables is much higher under previous cannabis use. This is to a large extent driven by the different coefficient estimates on accessibility factors under the two cannabis use states that we showed in previous section. We further observe a larger marginal average effect of proneness under previous cannabis use which is a result of the non-linear transformation of in the probit model.

Another approach to evaluate the importance of the three factors is to investigate the effects of changes in the three factors on hard drug initiation. We consider a number of different scenarios, for example an increase or decrease in drug supply or prices. We begin again by examining the implications for the marginal predictive probabilities of hard drug initiation $\operatorname{Pr}\left(h_{j, n+1}=1 \mid \mathbf{c}, \mathbf{h}, \mathbf{W}\right)$ where $j=0,1$ refers to assumed previous cannabis use. The results are given in the second and third columns of Table 4. To estimate how these scenarios would affect the overall hard drug initiation in the population (with and without previous cannabis use), we also report the predictive conditional probability of hard drug initiation $\operatorname{Pr}\left(h_{n+1}=1 \mid c_{n+1}, \mathbf{c}, \mathbf{h}, \mathbf{W}\right)$ under the different scenarios in the last column of Table 4. This measure is based on the joint probability of cannabis and hard drug initiation and takes into account a subject's probability of previous cannabis use (see Appendix A).

We first consider a scenario of a change in the availability of hard drugs (measured by seizure and prevalence). Our results suggest that a $50 \%$ decrease in availability would reduce the overall probability of hard drug uptake from 0.135 to 0.117 (see last column of Table 4). As expected, an higher accessibility will have the opposite effect, increasing the overall probability from 0.135 to 0.156 . While changes in accessibility to hard drugs have almost no effect on non-users of cannabis (both in estimation model and prediction results), the impact on cannabis users is non-trivial.

We further examine a scenario for changes in the proneness variables. A reduction proneness factors that lead to early alcohol initiation and school drop-outs would reduce the overall 


\begin{tabular}{lrrr}
\hline \multicolumn{4}{c}{ Predicted Hard Drug Uptake for Different Scenarios } \\
& No Cannabis & Cannabis & Overall \\
\hline Base & 0.077 & 0.363 & 0.135 \\
& & & \\
high hard drug seizure and prevalence & 0.081 & 0.550 & 0.156 \\
low hard drug seizure and prevalence & 0.075 & 0.233 & 0.117 \\
more early alcohol use and leave school & 0.082 & 0.381 & 0.163 \\
no early alcohol use and leave school & 0.068 & 0.352 & 0.087 \\
high can prevalence & - & - & 0.149 \\
low can prevalence & - & - & 0.120 \\
high can price & - & - & 0.144 \\
low can price & - & - & 0.123 \\
high constant gateway effect & - & - & 0.183 \\
lower constant gateway effect & - & - & 0.091 \\
\hline
\end{tabular}

Table 4: Means of the predicted probabilities of hard drug initiation for different scenarios based on the extended gateway model.

probability of hard drug initiation to 0.087 . An increase in proneness to drug use that would be reflected in a $50 \%$ increase in the early alcohol use and school drop outs would increase the probability to 0.163 .

Given the link between cannabis and hard drug use, however, an interesting policy question is how changes in the accessibility to cannabis would affect hard drug initiation. By employing the conditional predictive probability of hard drug uptake (instead of the marginal predictive probability), we can explore this question. Lowering the prevalence of cannabis use by $50 \%$ (everything else held constant) would decrease the probability of hard drug uptake from 0.135 to 0.120 , while a $50 \%$ increase in the prevalence raises the probability to 0.149 . Changes in the cannabis price, on the other hand, only have a very small effect on the overall probability of hard drug uptake. Lastly, we consider a change in the strength of the direct effect of previous cannabis use. For instance, a policy aiming at separating the markets for cannabis and hard drugs (in line with what is found in the Netherlands), may reduce the effect previous cannabis use has on hard drug initiation if the policy change reduces the social interaction between different groups drug users (mechanism 4 of those listed in section 1). In contrast, a more profound gateway effect than the one estimated here, is an alternative possible future scenario as improved cultivation techniques have resulted in cannabis with higher THC levels, i.e. more potent cannabis. More potent cannabis could imply stronger addiction to the drug and more craving also for other, and 
stronger, drug experiences (mechanism 1 in section 1). According to the World Drug Report 2007 (UN, 2007), the prevalence of this high-potent cannabis has increased substantially in recent years. The last two rows of Table 4 show that a $50 \%$ decrease/increase in the coefficient on previous cannabis use are the scenarios with the largest effect on hard drug initiation. A decrease will reduce the overall probability of hard drug uptake from 0.135 to 0.091 and an increase will raise it to 0.183 .

\section{Sensitivity Analysis}

In this section we present selected results from the prior-posterior and predictive analysis from different specifications of the extended gateway model. We consider different frequency definitions of the cannabis and hard drug variables, vary which types of hard drugs to include in the hard drug variable and employ different specifications of the variable vector in the hard drug equation. The purpose is 1) to check the robustness of our findings from the previous section regarding the direct and extended gateway effects and the importance of accessibility, proneness and previous cannabis use for hard drug initiation; and 2) to aid the interpretation of our results with respect to the transmission channels of previous cannabis use.

In Tables 5 and 6 we provide results from the fitting and the predictive analysis of the

\begin{tabular}{|c|c|c|c|c|}
\hline \multicolumn{5}{|c|}{ Some Results for Various Specifications of Variable Vectors } \\
\hline & \multirow{2}{*}{\multicolumn{2}{|c|}{$\begin{array}{c}\text { Spec 1 } \\
\text { Hard uptake }\end{array}$}} & \multicolumn{2}{|c|}{ Spec 2} \\
\hline & & & Hard & ptake \\
\hline & no can & can & no can & can \\
\hline Country & $0.03(0.24)$ & $0.23(0.16)$ & $0.05(0.24)$ & $0.25(0.19)$ \\
\hline Oslo & $0.48(0.34)$ & $-0.73(0.38)$ & $0.27(0.36)$ & $-1.12(0.50)$ \\
\hline Obtain & $0.21(1.09)$ & $0.03(0.91)$ & $-0.37(1.10)$ & $-0.18(1.13)$ \\
\hline seizure & $-0.05(0.78)$ & $2.38(0.92)$ & $0.25(0.83)$ & $2.78(1.19)$ \\
\hline Prevalence & $-0.16(1.34)$ & $2.83(1.84)$ & $0.03(1.36)$ & $2.55(1.99)$ \\
\hline Non-Western & $-0.08(0.22)$ & $-0.10(0.21)$ & $-0.22(0.24)$ & $0.37(0.27)$ \\
\hline Amph. Price & $0.49(0.50)$ & $-0.08(0.37)$ & $0.54(0.55)$ & $-0.18(0.50)$ \\
\hline Can. Use & 0.93 & $0.48)$ & 1.24 & $0.57)$ \\
\hline Intercept & -2.91 & $0.54)$ & -2.81 & $0.57)$ \\
\hline smoking & inc & ded & incl & ded \\
\hline Other* & inc & ded & incl & ded \\
\hline
\end{tabular}

Table 5: Specifications 1 and 2 refer to the main model with the different frequency definitions of the depended variables, freq11 and freq22. *All specifications include the standard proneness and demographic variables from the main estimation. 
extended gateway model under two alternative frequency definitions of the cannabis and hard drug variables, $c_{i}$ and $h_{i}$. So far we have defined a cannabis user $\left(c_{i}=1\right)$ as a person that has used cannabis at least 5 times (and $c_{i}=0$ otherwise) and a hard drug user $\left(h_{i}=1\right)$ as a person who has used hard drugs at least once (and $h_{i}=0$ otherwise). Under Specification 1 we now define a person as a cannabis user (hard drug users) if he/she has used cannabis (any hard drug) at least once. Under Specification 2 we define a person as cannabis user (hard drug user) if he/she has used cannabis (any hard drug) at least five times. We find that the gateway coefficient varies from 0.93 for Specification 1 to 1.24 for Specification 2 compared to 1.20 in the main model. The predicted probabilities for these specifications differ somewhat more from those estimated for the main model (Table 3). However, the differences are consistent with the frequencies in the raw data (see Table 11 in Appendix B). As before, we find that accessibility and proneness factors contribute significantly to hard drug uptake in addition to previous cannabis use, composed of a direct effect and a extended effect via the accessibility variables.

A comparison of the coefficient estimates (posterior means) from the fitting of Specifications 1 in Table 5 to those from the main model in Table 2 yields some interesting insights and possible interpretations regarding the transmission channels of previous cannabis use on hard drug uptake. First, we find that the posterior mean of the coefficient on previous cannabis use decreases from 1.20 to 0.93 if we include also low frequent cannabis user (life time use between one and four times) into the group of cannabis users. This observations is coherent with the fact that one would expect the strength of the transmission channels 1 and 4 to be lower for incidental and low frequency cannabis users as they have not yet developed a strong psychological or physiological desire for harder drugs and are less knowledgeable about the drug market. Further, we don't see large changes in the coefficients on the accessibility variables. This observations is consistent with the transmission channels 2 and 3 which claim that having tried cannabis will lower the threshold and perceived risk of using a harder drug. Using cannabis only once or twice is sufficient for a subject to pass the threshold to illegal drugs and to be less concerned about their potential harmful effects, increasing the probability to take up hard drugs given the opportunity. 
An alternative way to explore the robustness of our findings with respect to the definition of the hard drug use variable is to consider more narrowly defined hard drug use variables. We have redone the analysis using two alternative hard drug variables: use of any hard drug other than ecstasy (specification 3); amphetamine use (specification 4). The definition of the first variable is motivated by the fact that ecstasy is sometimes viewed as a softer "party drug", while the second variable will allow us to look at the effects on a single hard drug variable, which on average is the hard drug with the lowest starting age and the only one for which we have price data. The estimates for the predictive analysis are given in Table 6 . The results for hard drug use without ecstasy use are roughly comparable to those in the main model. This is not too surprising as the vast majority of ecstasy users reports the use of other hard drugs. This is also reflected in the similar estimates of the model parameters, with an almost identical estimate for the constant gateway effect (see Table 14 in the appendix). As expected, the gateway coefficient for amphetamine use is lower (about three quarters of the that in the main model) which is reflected in lower hard drug probability under previous cannabis use. Again, the partial probabilities suggest the largest effect from the constant gateway effect, followed by that of accessibility and proneness.

\begin{tabular}{lrrrrrrrrr}
\hline & \multicolumn{1}{c}{ Predictive Probabilities Under Various Specifications } & \multicolumn{2}{c}{ Spec 4 } \\
& \multicolumn{2}{c}{ Spec 1 } & \multicolumn{2}{c}{ Spec 2 } & \multicolumn{2}{c}{ Spec 3 } & \multicolumn{2}{c}{ Spec } \\
& no can & can & no can & can & no can & can & no can & can \\
\hline Total & & & & & & & & \\
w/o Access & 0.056 & 0.221 & 0.035 & 0.246 & 0.064 & 0.363 & 0.055 & 0.274 \\
w/o Prices & 0.036 & 0.091 & 0.037 & 0.141 & 0.031 & 0.154 & 0.048 & 0.123 \\
w/o Gateway & 0.038 & 0.237 & 0.023 & 0.280 & 0.039 & 0.314 & 0.030 & 0.229 \\
w/o Proneness & 0.019 & 0.069 & - & -0.051 & - & 0.098 & - & 0.112 \\
\hline
\end{tabular}

Table 6: Means of predictive probabilities of hard drug initiation for various specifications of the extended gateway model.

We have also considered two alternative sets of explanatory variables. Firstly, we acknowledge the potential endogeneity issues with respect to some of our control variables, in particular for the lifetime smoking and the obtain variables, which may bias our results. To address this concern, we have estimated the extended gateway model without these variables (Specification 5 , full set of results are available upon request). Again we find that our results do no change 
dramatically compared to those in the main model. As expected, the exclusion of the smoking variable from the proneness vector $\mathbf{w}_{p, i}$ leads to a smaller overall effect of proneness on hard drug uptake. We do observe a higher total predictive probability of hard drug uptake for cannabis users under this specification.

Secondly, we have addressed the issue of early cannabis use. It has been suggested in the literature that starting with cannabis very young could reinforce the gateway effect. Animal studies (Ellgren et al., 2007, Fergusson et al., 2006) and twin studies examining same-sex twin pairs discordant for early cannabis initiation (see for example Lynskey et al., 2006) seem to support this notion. We therefore consider a specification that extends our main model by including an additional indicator for early cannabis use in the hard drug model (Specification 6). The indicator is 1 if the respondents report to have used cannabis before they turn 16 . We find that early cannabis use has a positive effect on hard drug uptake (0.71). Interestingly we now observe a lower mean of the posterior distribution of previous cannabis use of 0.60 compared the estimate of 1.20 for the main specification (Table 2). One might speculate that this implies that the estimated direct gateway effect is to a large extent driven by early cannabis use. However, the early use variable might be associated with endogeneity issues as we cannot rule out that early use is at least partially driven by unobserved proneness factors that also drive hard drug uptake. If, on the other hand, the early use coefficient reflects mainly unobserved proneness that for some reason has not been captured by our joint modeling, then this would imply a smaller gateway effect than the one presented in Table 2. Finally, we should note that the inferences of the other coefficients remain fairly stable and we again observed an extended gateway effect.

\section{Discussion}

Extensive use of hard drugs is associated with substantial health risks and adverse consequences for educational achievements, social relationships, employment and careers. Most countries therefore rely on a drug policy aiming to reduce the use and misuse of substances such as cocaine, amphetamine, ecstasy and heroin and many uphold a strict cannabis regime to lower hard drug 
use. However, as pointed out in the recent literature (e.g. Fergusson et al., 2006, Kandel et al., 2006), the mechanisms which could drive the relationship between previous cannabis use and hard drug use are not well understood and require empirical investigations. In addition, the efficacy of drug policy to reduce hard drug use will depend also on the role of accessibility and proneness factors. Thus, a better understanding of the factors influencing hard drug uptake is needed to improve drug policy means.

Using data from a representative sample of young adults (21-30 years)in Norway and a novel modeling approach, this paper examines the influence of previous cannabis use, accessibility and proneness factors on the probability of hard drug uptake. To the best of our knowledge, this paper is the first attempt in the literature to examine the three hypotheses simultaneously and to quantify the relative importance of the three effects on the probability of hard drug uptake. Our results show that all three factors matter, with the effect of previous cannabis use dominating the effects from the observed accessibility and proneness factors. Our predictive analysis suggests that, for a random person, the probability of taking up hard drugs increases from roughly 8 per cent, if he/she has not previously used cannabis, to 36 per cent for cannabis users. Both proneness and accessibility have larger effects for cannabis users than for non-cannabis users, with the overall effects (measured as marginal average effects) from accessibility being more important than those of proneness. This is partly due to the fact that even though many of the proneness variables proved important, relatively few individuals reported problems with police and parents or started to drink alcohol before they turned 13. As shown in the sensitivity analysis, our general findings are robust across various model specifications (e.g. alternative definitions of the dependent variables and sets of covariates).

Our extended gateway model for hard drug initiation sheds some light on the different transmission channels of cannabis use on hard drug initiation that have been proposed in the literature. We find a positive coefficient on previous cannabis use in our main model specification. The coefficient decreases if we include "incidental" cannabis users (having used cannabis less than 5 times). These results are in line with channel 1 that suggests (i) cannabis causes a psychological and/or physiological desire for stronger drug experiences reflected in a positive 
constant gateway effect and (ii) that the effect increases with a higher frequency of cannabis use. Further, we observe that the coefficient estimates of the accessibility variables on hard drug initiation depend on previous cannabis use. Also, our estimated coefficients on accessibility show little variation across the models with different frequency specifications of the drug use variables. This indicates that once a subject has passed the threshold to illegal drug use, the frequency of use is not of great importance for the accessibility effects as one would expect from the second and third transmission channels. The channels suggest a lower credibility in warnings and reduced costs of trying another drug after personal illegal drug experience, and imply a lower threshold for hard drug use after cannabis has been initiated. Finally, the positive coefficient on previous cannabis use and to some degree the dependence of the coefficients on accessibility on previous cannabis use may also (partially) reflect the fourth transition channel which refers to a better knowledge of the hard drug market and more opportunities of hard drug use for cannabis users. The raw data strongly suggests that there are differences between cannabis users and non-users with regard to reported obtainability of hard drugs, which is in line with the findings in Wagner and Anthony (2002). Table 10 presented in Appendix B shows that only 31, 23, 19 and 29 per cent of non-users claim to be able to obtain amphetamine, cocaine, heroin and ecstasy, respectively, within three days. The corresponding numbers for cannabis users are 62 , 57, 33 and 48 per cent. Also, more cannabis users report that they have been offered hard drugs (80 vs $26 \%$ ). Probable endogeneity problems restrain us from including these variables in the estimations.

With regard to possible policy implications, the finding of a substantial gateway effect suggests that any intervention successful in reducing cannabis use will also reduce the uptake of hard drugs. Previous cannabis use is the single most important variable for the increased probability of starting to use drugs like amphetamine and cocaine. Further, for any given level of cannabis use, means for weakening the transition mechanisms should be of interest to policy makers. For instance, a separation of markets for cannabis and hard drugs may prove effective in reducing hard drugs uptake if that would reduce the influence hard drug users and knowledge of those markets might have on current cannabis-only users. Increased credibility of health warnings, 
something which might be achieved by providing more drug specific information instead of focusing on illegal drugs in general, is another means to weaken the transition mechanisms. The recent increase in the prevalence of high-potent cannabis may, on the other hand, strengthen the effect of cannabis use. Further, our findings regarding observed proneness factors indicate that intervention directed against high risk groups, e.g. early detection and adequate interference towards subgroups with high prevalence of the problem indicators (early alcohol debut, low educational achievements, problems with parents and police), will reduce hard drug initiation. 


\section{References}

Albert J, Chib S. 1993. Bayesian Analysis of Binary and Polychotomous Response Data. Journal of the American Statistical Association 88: 669-779.

Beenstock M, Rahav, G. 2002. Testing Gateway Theory: do cigarette prices affect illicit drug use? Journal of Health Economics 21: 679-698.

Bretteville-Jensen AL, Biorn E. 2004. Do prices count? A micro-econometric study of illicit drug consumption based on self-reported data. Empirical Economics 29(3): 673-695.

Bretteville-Jensen AL, Melberg HO. and Jones, A. Sequential patterns of drug use initiation can we believe in the gateway theory? The B.E. Journal of Economic Analysis 83 Policy (Contributions) 8 (2), Article 1.

Chib S. 2007. Analysis of Treatment Response Data without the joint distribution of Potential Outcomes. Journal of Econometrics 140: 401-412.

Chib S, Greenberg E. 1995. Understanding the Metropolis-Hastings algorithm. American Statistician 49: 327-35.

Chib S, Greenberg E. 1998. Analysis of multivariate probit models. Biometrika 85: 347-361.

Chib S, Jacobi L. 2007. Modeling and Calculating the Effect of Treatment at Baseline from Panel Outcomes. Journal of Econometrics 140: 781-801.

Contoyannis, P., Jones, AM. 2004. Socio-economic status, health and lifestyle. Journal of Health Economics, 23: 965-995.

DeSimone J. 1998. Is marijuana a gateway drug? Eastern Economic Journal 24: 149-164.

Ellgren M, Spano, SM, Hurd YL. 2007. Adolescent cannabis exposure alters opiate intake and opioid limbic neuronal populations in adult rats. Neuropsychopharmacology 32: 607-615.

EMCDDA. 2007. Annual report 2007: The state of the drugs problem in Europe. European Monitoring Centre for Drug use and Drug Addiction, Lisbon, Portugal.

Fergusson DM, Boden JM and Horwood LJ. 2006. Cannabis use and other illicit drug use: testing the cannabis gateway hypothesis. Addiction 101: 556-569.

Greene, WH. 2002. Econometric Analysis (fifth edition), Prentice Hall, New Jersey.

Kandel DB. 1975. Stages in adolescent involvement in drug use. Science 190: 912-914.

Kandel DB (ed.) 2002. Stages and pathways of drug involvement: Examining the gateway hypothesis. Cambridge University Press: Cambridge, England.

Kandel DB, Yamaguchi K, Klein LC. 2006. Comments to Fergusson et al. Addiction 101: 470-472.

Lynskey MT, Vink JM, Boomsma DI. 2006. Early onset of cannabis use and progression to other drug use in a sample of Dutch twins. Behavior Genetics 36(2): 195-199.

Maddala, GS. 1983. Limited-dependent and qualitative variables in econometrics, Cambridge University Press, Cambridge. 
Melberg HO, Bretteville-Jensen AL, Jones A. Is cannabis a gateway to hard drugs? Empirical Economics, forthcoming.

Morral AR, McCaffrey DF, Paddock S.M. 2002. Reassessing the marijuana gateway effect. Addiction 97: 1493-1504.

Nutt D, King LA, Saulsbury W, Blakemore C. 2007. Development of a rational scale to assess the harm of drugs of potential misuse. The Lancet 369(9566): 1047-1053.

Pudney S. 2003. The road to ruin? Sequences of initiation to drug use and crime in Britain. Economic Journal 113: 182-198.

Reinarman C, Cohen PDA, Kaal HL. 2004. The limited relevance of drug policy: cannabis in Amsterdam and in San Francisco, American Journal of Public Health 94(5): 836-842.

Skog OJ. 1992. The validity of self-reported drug use. British Journal of Addiction 87: 539548.

Van Ours, JC. 2003. Is cannabis a stepping-stone for cocaine? Journal of Health Economics 22: $539-554$.

Van Ours, JC., Williams J. 2007. Cannabis prices and dynamics of cannabis use. Journal of Health Economics 26: 578596.

Wagner A, Anthony JC. 2002. Into the World of Illegal Drug Use: Exposure Opportunity and Other Mechanisms Linking the Use of Alcohol, Tobacco, Marijuana and Cocaine. American Journal of Epidemiology 155: 918-925.

World Drug Report 2007: Volume 1, Analysis. Office on Drugs and Crime, United Nations Publication, 2007 


\section{Appendix A: Model Estimation}

\subsection{Prior Specification}

To specify the hyper-parameters in the prior distribution given by equation (3.7) for the estimation we exploit data from the 2002 survey on drug use. We first fit the model described in Section 3.1 without the nonwest and hard drug price variables in the vector $\mathbf{w}_{a h, i}$ and without the cannabis price variable in $\mathbf{w}_{a c, i}$ as we have insufficient information for these in the 2002 data. Otherwise the covariate vectors are defined as specified in Section 3.1. The means of the prior distributions are centered at zero to express our apriori ignorance and the prior variances are set to 5 for the slope parameters and 1 for the correlation parameters to ensures sufficient prior flexibility. The fitting leads to the following posterior means for the coefficients in the hard drug and cannabis equations:

$$
\begin{aligned}
\hat{\boldsymbol{\beta}}_{0,2002} & =(-0.11,-0.36,-1.04,1.61,-0.28,0,0) \\
\hat{\boldsymbol{\beta}}_{1,2002} & =(0.66,-0.43,-1.40,2.21,1.0,0,0), \\
\hat{\boldsymbol{\alpha}}_{2002} & =(-1.78,1.29,0.78,0.18,0.32,-0.24,0.62,0.44,0,0.10,0.15), \\
\hat{\boldsymbol{\gamma}}_{2002} & =(-1.22,-0.35,0.04,0.08,0.40,0,0,0.64,0.47,0.48,-0.19,0.06,0.62,0,0.25,0.14) .
\end{aligned}
$$

We take these posterior means to reflect our updated assumptions about the prior means in the cannabis and hard drug models after seeing the 2002 data:

$$
\boldsymbol{b}_{00}=\hat{\boldsymbol{\beta}}_{0,2002}, \boldsymbol{b}_{10}=\hat{\boldsymbol{\beta}}_{1,2002}, \boldsymbol{a}_{0}=\hat{\boldsymbol{\alpha}}_{2002}, \boldsymbol{g}_{0}=\hat{\boldsymbol{\gamma}}_{2002}
$$

The values in $\boldsymbol{b}_{00}$ and $\boldsymbol{b}_{10}$ refer to the accessibility variables in $\mathbf{w}_{a h, i}, \boldsymbol{a}_{0}$ to the covariate vector $\left\{\right.$ const $\left.\sim c_{i} \sim \mathbf{w}_{p, i} \sim \mathbf{w}_{d, i}\right\}$ and $\boldsymbol{g}_{0}$ to the covariate vector $\left\{\right.$ const $\sim c_{i} \sim \mathbf{w}_{a c, i} \sim \mathbf{w}_{p, i} \sim$ $\left.\mathbf{w}_{d, i}\right\}$. For example, our specification of $\boldsymbol{b}_{00}$ and $\boldsymbol{b}_{10}$ suggest that accessibility factors affect hard drug initiation, and do so in a different way depending on previous cannabis use. The lower prior means for the seizure and prevalence variables for subjects without previous cannabis use $(1.61,-0.28)$ compared to those with prior use $(2.21,1.0)$ express a prior belief that previous cannabis affects hard drug initiation via transmission channels 2 and 3 . The positive prior mean of 1.29 on the gateway coefficient assumes that previous cannabis use affects hard drug uptake directly (via transmission channels 1 and/or 4). Note that we have set the prior means of the non-western and price variables at zero since we cannot update our prior ignorance based on the 2002 data.

To ensure that our prior assumptions can be updated based on the new data via the likelihood of the 2006 data, we again formulate flexible priors around these specified means. In particular, we set the variances for all slope coefficient at 2. Based on the 2002 data analysis, we center the prior distributions of the correlation parameters $\left(\rho_{0}, \rho_{1}\right)$ at 0.15 and 0 with a variance of 1 .

\subsection{Algorithm for Prior-Posterior Analysis}

Due to the structure of the likelihood function, the posterior distribution $\pi(\boldsymbol{\theta} \mid \mathbf{h}, \mathbf{c}, \mathbf{W})$ of the vector of model parameters $\boldsymbol{\theta}=\left(\boldsymbol{\beta}_{0}, \boldsymbol{\beta}_{1}, \boldsymbol{\alpha}, \boldsymbol{\gamma}, \rho_{0}, \rho_{1}\right)$ is not readily estimable via Markov Chain Monte Carlo (MCMC) methods. We can however, exploit the ideas in Albert and Chib (1993) and include the latent drug intake variables in the parameter space and work with the augmented posterior distribution $\pi\left(\boldsymbol{\theta}, \mathbf{c}_{i}^{*}, \mathbf{h}_{i}^{*} \mid \mathbf{h}, \mathbf{c}, \mathbf{W}\right)$, which is given by the expression

$$
\pi\left(\boldsymbol{\theta}, \mathbf{h}_{i}^{*}, \mathbf{c}_{\mathbf{i}}^{*} \mid \mathbf{h}, \mathbf{c}, \mathbf{W}\right) \propto \pi(\boldsymbol{\theta}) p\left(\mathbf{h}, \mathbf{c}, \mathbf{h}_{\mathbf{i}}{ }^{*}, \mathbf{c}_{\mathbf{i}}{ }^{*} \mid \mathbf{W}, \boldsymbol{\theta}\right)
$$


where the joint likelihood of the augmented parameter space $p\left(\mathbf{h}, \mathbf{c}, \mathbf{h}_{j i}^{*}, \mathbf{c}_{\mathbf{i}}{ }^{*} \mid \mathbf{W}, \boldsymbol{\theta}\right)$ takes the form

$$
\prod_{i=1}^{N} \mathcal{N}_{2}\left(h_{j i}^{*}, c_{i}^{*} \mid \mathbf{W}_{j i}^{\prime} \boldsymbol{\delta}, \boldsymbol{\Omega}_{j}\right)\left[I\left\{c_{i}^{*} \leq 0\right\}^{1-c_{i}}+I\left\{c_{i}^{*}>0\right\}^{c_{i}}\right]\left[I\left\{h_{j i}^{*} \leq 0\right\}^{1-h_{j i}}+I\left\{h_{j i}^{*}>0\right\}^{h_{j i}}\right]
$$

$h_{i}=h_{0 i}$ if $c_{i}=0$ and $h_{i}=h_{1 i}$ if $c_{i}=1$. The prior distribution is given by expression (3.7). The resulting joint posterior distribution is of a type that can be efficiently processed by MCMC methods. We propose the following six step MCMC chain to generate draws from the posterior distribution. We let $\mathbf{c}=\left(\mathbf{c}_{0}, \mathbf{c}_{1}\right)$ where $\mathbf{c}_{0}=\left(c_{i}: i \in N_{0}\right)$ and $\mathbf{c}_{1}=\left(c_{i}: i \in N_{1}\right)$ represent the cannabis observations under the two possible cannabis states; with a similar convention for the latent cannabis intake is $\mathbf{c}^{*}=\left(\mathbf{c}_{0}^{*}, \mathbf{c}_{1}^{*}\right)$. We propose the following algorithm to generate draws from the posterior distribution for the extended gateway model proposed in Section 3.1-2. The algorithm is run for $\mathrm{M}$ (for example $M=10,000$ ) iterations. A burn-in phase of 1,000 iterations is used to ensure that MCMC chain has converged to the posterior distribution of interest.

\section{MCMC Algorithm:}

1. Initialize $\boldsymbol{\beta}_{j}, \boldsymbol{\alpha}, \boldsymbol{\gamma}, \rho_{j},\left\{c_{i}^{*}\right\}$ for $j=0,1$

2. Sample $h_{j, i}^{*} \mid h_{j i}, c_{i}, c_{i}^{*}, \boldsymbol{\beta}, \boldsymbol{\alpha}, \boldsymbol{\gamma}, \rho$ for all subjects in $N_{1}$ from the conditional truncated normal $N\left(h_{j, i}^{*} \mid \mathbf{w}_{1 h, i}^{\prime} \boldsymbol{\beta}_{j}+\mathbf{w}_{2 h, i}^{\prime} \boldsymbol{\alpha}+\rho_{j}\left(c_{i}^{*}-\mathbf{w}_{c, i}^{\prime} \gamma\right), 1-\rho_{j}^{2}\right)\left[I\left\{h_{j i}^{*} \leq 0\right\}^{1-h_{j i}}+I\left\{h_{j i}^{*}>0\right\}^{h_{j i}}\right]$

3. Sample $c_{i}^{*} \mid h_{j i}, c_{i}, h_{i}^{*}, \boldsymbol{\beta}_{j}, \boldsymbol{\gamma}, \rho_{j}$ from the conditional truncated normal

$$
N\left(c_{i}^{*} \mid \mathbf{w}_{c, i}^{\prime} \boldsymbol{\gamma}+\rho_{j}\left(h_{i}^{*}-\mathbf{w}_{1 h, i}^{\prime} \boldsymbol{\beta}_{j}-\mathbf{w}_{2 h, i}^{\prime} \boldsymbol{\alpha}\right), 1-\rho_{j}^{2}\right)\left[I\left\{c_{i}^{*} \leq 0\right\}^{1-c_{i}}+I\left\{c_{i}^{*}>0\right\}^{c_{i}}\right] .
$$

4. Sample $\boldsymbol{\gamma} \mid \mathbf{h}, \mathbf{c}, \mathbf{h}^{*}, \mathbf{c}^{*}, \boldsymbol{\beta}, \boldsymbol{\alpha}, \rho$ from $\mathcal{N}_{k}(\boldsymbol{\gamma} \mid \hat{\boldsymbol{\gamma}}, \mathbf{G})$, where

$\hat{\gamma}=\mathbf{G}\left[\mathbf{G}_{0}^{-1} \mathbf{g}_{0}+\sum_{i \in N} \mathbf{w}_{c, i} \sigma_{j}^{-2}\left(c_{i}^{*}-\rho_{j} \hat{h}_{i}^{*}\right)\right]$,

$\mathbf{G}=\left[\mathbf{G}_{0}^{-1}+\sum_{i \in N} \mathbf{w}_{c, i} \sigma_{j}^{-2} \mathbf{w}_{c, i}^{\prime}\right]^{-1}$,

with $\sigma_{j}^{2}=\left(1-\rho_{j}^{2}\right)$ and $\hat{h}_{i}^{*}=h_{i}^{*}-\mathbf{w}_{1 h, i}^{\prime} \boldsymbol{\beta}_{j}-\mathbf{w}_{2 h, i}^{\prime} \boldsymbol{\alpha}$.

5. Sample $\boldsymbol{\beta}_{j} \mid \mathbf{h}, \mathbf{c}, \mathbf{h}^{*}, \mathbf{c}_{1}^{*}, \boldsymbol{\gamma}, \rho$ from $\mathcal{N}_{p}(\boldsymbol{\beta} \mid \hat{\boldsymbol{\beta}}, \mathbf{B})$, where

$$
\begin{aligned}
& \hat{\boldsymbol{\beta}}_{j}=\mathbf{B}_{j}\left[\mathbf{B}_{j, 0}^{-1} \mathbf{b}_{j, 0}+\sum_{i \in N_{j}} \mathbf{w}_{1 h, i} \sigma_{j}^{-2}\left(h_{i}^{*}-\mathbf{w}_{2 h, i}^{\prime} \boldsymbol{\alpha}-\rho_{j} \hat{c}_{i}^{*}\right)\right], \\
& \mathbf{B}_{j}=\left[\mathbf{B}_{j, 0}^{-1}+\sum_{i \in N_{j}} \mathbf{w}_{1 h, i} \sigma_{j}^{-2} \mathbf{w}_{1 h, i}^{\prime}\right]^{-1} \text { with } \sigma_{j}^{2}=\left(1-\rho_{j}^{2}\right), \\
& \text { and } \hat{c}_{i}^{*}=c_{i}^{*}-\mathbf{w}_{c, i}^{\prime} \boldsymbol{\gamma}
\end{aligned}
$$

6. Sample $\boldsymbol{\alpha} \mid \mathbf{h}, \mathbf{c}, \mathbf{h}^{*}, \mathbf{c}_{1}^{*}, \boldsymbol{\gamma}, \rho$ from $\mathcal{N}_{m}(\boldsymbol{\alpha} \mid \hat{\boldsymbol{\alpha}}, \mathbf{A})$, where

$$
\begin{aligned}
& \hat{\boldsymbol{\alpha}}=\mathbf{A}\left[\mathbf{A}_{0}^{-1} \mathbf{a}_{0}+\sum_{i \in N} \mathbf{w}_{2 h, i} \sigma_{j}^{-2}\left(h_{i}^{*}-\mathbf{w}_{1 h, i}^{\prime} \boldsymbol{\beta}_{j}-\rho_{j} \hat{c}_{i}^{*}\right)\right], \\
& \mathbf{A}=\left[\mathbf{A}_{0}^{-1}+\sum_{i \in N_{j}} \mathbf{w}_{2 h, i} \sigma_{j}^{-2} \mathbf{w}_{2 h, i}^{\prime}\right]^{-1} \text { with } \sigma_{j}^{2}=\left(1-\rho_{j}^{2}\right),
\end{aligned}
$$

7. Sample $\rho_{j} \mid \mathbf{h}_{j}, \mathbf{s}_{j}, \mathbf{h}_{j}^{*}, \mathbf{s}_{j}^{*}, \boldsymbol{\beta}_{j}, \boldsymbol{\gamma}$, for $j=0,1$ from an MH step described below.

8. Goto 2 
Since the full conditional distribution of the correlation coefficient $\rho_{j}$ is not tractable, we update the parameters using the Metropolis Hastings algorithm. Following Chib and Greenberg $(1995,1998)$ we generate proposal values $\rho_{j}^{\prime}$ from a tailored student-t density $t_{\nu}\left(\mu_{j}, V_{j}\right)$ where $\mu_{j}$ is the approximate mode of

$$
\ln \left(\prod_{I \in I_{j}} \mathcal{N}\left(h_{i}^{*}, c_{i}^{*} \mid \mathbf{W}_{i} \boldsymbol{\delta}_{j}, \boldsymbol{\Omega}_{j}\right)\right.
$$

and $V$ is the inverse Hessian of this density evaluated at $\mu_{j}$. We accept the proposal value with probability of move $\alpha_{j}$ where

$$
\alpha_{j}=\min \left\{1, \frac{\pi\left(\rho_{j}^{\prime}\right) \prod_{i \in I_{j}} \mathcal{N}\left(h_{i}^{*}, c_{i}^{*} \mid \mathbf{W}_{i} \boldsymbol{\delta}_{j}, \boldsymbol{\Omega}_{j}\right) \times t_{\nu}\left(\rho_{j} \mid \mu_{j}, V_{j}\right)}{\pi\left(\rho_{j}\right) \prod_{i \in I_{j}} \mathcal{N}\left(h_{i}^{*}, c_{i}^{*} \mid \mathbf{W}_{i} \boldsymbol{\delta}_{j}, \boldsymbol{\Omega}_{j}\right) \times t_{\nu}\left(\rho_{j}^{\prime} \mid \mu_{j}, V_{j}\right)}\right\}
$$

\subsection{Extended Algorithm for Predictive Analysis}

The marginal predictive probabilities of hard drug use $\operatorname{Pr}\left(h_{j, n+1}=1 \mid \mathbf{c}, \mathbf{h}, \mathbf{W}\right), j=0,1$ can be computed based on the predictive distributions of the latent hard drug intake. To generate draws from $p\left(h_{j, n+1}^{*} \mid \mathbf{h}, \mathbf{s}, \mathbf{W}, \mathbf{w}_{h, n+1}\right)$ we exploit the fact that the conditional predictive distribution $p\left(h_{j, n+1}^{*} \mid \mathbf{h}, \mathbf{s}, \mathbf{W}, \mathbf{w}_{h, n+1}, \boldsymbol{\beta}_{j}, \boldsymbol{\alpha}\right)$ is of a known form. To obtain the marginal predictive distribution we integrate numerically over the parameters using the corresponding posterior distribution and over $\mathbf{w}_{h, n+1}$ using the empirical distribution of the data. This can be done in a straight forward manner by extending the model fitting algorithm, that generates draws of the model parameters from the posterior distribution, by the following steps at each iteration $g$ of the algorithm:

- Sample $\mathbf{w}_{1 h, n+1}^{(g)}$ and $\mathbf{w}_{2 h, n+1}^{(g)}$ from the full set of covariates

- Compute $\operatorname{Pr}\left(h_{j, n+1}^{*(g)}>0 \mid \mathbf{w}_{1 h, n+1}^{(g)}, \boldsymbol{\beta}_{j}^{(g)}, \boldsymbol{\alpha}^{(g)}\right)$ where $h_{j, n+1}^{*(g)} \sim \mathcal{N}\left(h_{j, n+1}^{*(g)} \mid \mathbf{w}_{1 h, i}^{g^{\prime}} \boldsymbol{\beta}_{j}^{(g)}+\mathbf{w}_{2 h, i}^{g^{\prime}} \boldsymbol{\alpha}^{(g)}, 1\right)$ and $\boldsymbol{\beta}_{j}^{(g)}$ and $\boldsymbol{\alpha}^{(g)}$ are the parameter values at the current $\mathrm{g}^{\prime}$ th iteration of the MCMC algorithm

- Store values

The resulting draws $\left[h_{j, n+1}^{*^{1}}, h_{j, n+1}^{*^{2}}, \ldots, h_{j, n+1}^{*^{M}}\right]$ are from the marginal predictive distribution of latent hard drug intake. From these draws we can immediately compute the probabilities of hard drug intake as

$$
\operatorname{Pr}\left(h_{j, n+1}=1 \mid \mathbf{c}, \mathbf{h}, \mathbf{W}\right)=\frac{1}{M} \sum_{g=1}^{M} I\left[h_{j, n+1}^{*(g)}>0\right]
$$

To compute the partial predictive probabilities without accessibility effects etc we use the same approach and set the corresponding elements in $\boldsymbol{\beta}_{j}$ or $\boldsymbol{\alpha}$ to zero when drawing $h_{j, n+1}^{*(g)}$ from $\mathcal{N}\left(h_{j, n+1}^{*(g)} \mid \mathbf{w}_{h, i}^{g^{\prime}} \boldsymbol{\beta}_{j}^{(g)}+\mathbf{w}_{2 h, i}^{g^{\prime}} \boldsymbol{\alpha}^{(g)}, 1\right)$.

To compute the overall probability of hard drug use in Table 4, which is based on the conditional probability $\operatorname{Pr}\left(h_{j, n+1}=1 \mid c_{n+1}, \mathbf{c}, \mathbf{h}, \mathbf{W}\right)$, we use the following steps:

- Sample $\mathbf{w}_{1 h, n+1}^{(g)}, \mathbf{w}_{2 h, n+1}^{(g)}$ and $\mathbf{w}_{c, n+1}^{(g)}$ from the full set of covariates

- Draw $c_{n+1}^{*^{(g)}}$ from $\mathcal{N}\left(c_{n+1}^{*(g)} \mid \mathbf{w}_{c, i}^{g^{\prime}} \gamma^{(g)}, 1\right)$ and set $j=I\left[c_{n+1}^{*(g)}>0\right]$. 
- Compute $\operatorname{Pr}\left(h_{n+1}^{*(g)}>0 \mid \mathbf{w}_{1 h, n+1}^{(g)}, \boldsymbol{\gamma}^{(g)}, \boldsymbol{\alpha}^{(g)}, \rho_{j}, c_{n+1}^{*^{(g)}}=j\right)$ where $h_{n+1}^{*(g)} \sim \mathcal{N}\left(h_{j, n+1}^{*(g)} \mid \mathbf{w}_{h, i}^{g^{\prime}} \boldsymbol{\beta}_{j}^{(g)}+\right.$ $\left.\mathbf{w}_{2 h, i}^{g^{\prime}} \boldsymbol{\alpha}^{(g)}-\rho_{j}\left(c_{n+1}^{*(g)}-\mathbf{w}_{c, i}^{g^{\prime}} \gamma^{(g)}\right), 1-\rho_{j}^{2}\right)$

- Store values

The draws on $\operatorname{Pr}\left(h_{n+1}^{*^{(g)}}>0 \mid \mathbf{w}_{1 h, n+1}^{(g)}, \gamma^{(g)}, \boldsymbol{\alpha}^{(g)}, \rho_{j}, c_{n+1}^{*(g)}=j\right)$ can be summarized graphically and used to compute the mean and other statistics of the predictive distribution of the conditional probability of hard drug initiation $\operatorname{Pr}\left(h_{j, n+1}=1 \mid c_{n+1}, \mathbf{c}, \mathbf{h}, \mathbf{W}\right)$. 


\section{Appendix B: Additional Data Summaries and Estimation Re- sults}

\begin{tabular}{lcccc}
\hline & \multicolumn{2}{c}{ Distribution of Hard Drug Use } \\
Amphetamine & Cocaine & Ecstasy & Heroin \\
\# of hard drugs used & $(n=419)$ & $(n=419)$ & $(n=294)$ & $(n=63)$ \\
\hline 1 & $19 \%$ & $21 \%$ & $11 \%$ & $3 \%$ \\
2 & $31 \%$ & $30 \%$ & $19 \%$ & $8 \%$ \\
3 & $38 \%$ & $37 \%$ & $53 \%$ & $11 \%$ \\
4 & $12 \%$ & $12 \%$ & $17 \%$ & $78 \%$ \\
\hline
\end{tabular}

Table 7: Percentage of amphetamine, cocaine, ecstacy and heroin users that used 1, 2, 3 or 4 hard drugs.

\begin{tabular}{lcccccc}
\hline & \multicolumn{3}{c}{ Life Time Prevalence of Drug Use } & & \\
& Cannabis & Amphetamine & Cocaine & Ecstasy & Heroin & Hard Drugs \\
\hline Cannabis $(\mathrm{n}=1588)$ & $100 \%$ & $25.4 \%$ & $25.6 \%$ & $18.0 \%$ & $3.8 \%$ & $34.0 \%$ \\
Amphetamine $(\mathrm{n}=419)$ & $96.2 \%$ & $100 \%$ & $72.3 \%$ & $56.1 \%$ & $13.4 \%$ & - \\
Cocaine $(\mathrm{n}=418)$ & $97.1 \%$ & $72.5 \%$ & $100 \%$ & $54.0 \%$ & $13.9 \%$ & - \\
Ecstasy $(\mathrm{n}=293)$ & $97.6 \%$ & $80.2 \%$ & $77.1 \%$ & $100 \%$ & $17.7 \%$ & - \\
Heroin $(\mathrm{n}=63)$ & $96.8 \%$ & $88.9 \%$ & $92.1 \%$ & $82.5 \%$ & $100 \%$ & - \\
Hard Drugs $(\mathrm{n}=566)$ & $95.4 \%$ & $74.0 \%$ & $73.5 \%$ & $51.2 \%$ & $11.0 \%$ & $100 \%$ \\
\hline
\end{tabular}

Table 8: Life Time Prevalence of the use of cannabis and various hard drugs by drug use (in \%).

\begin{tabular}{lccccc}
\hline \multicolumn{7}{c}{ Distribution of Problem Variables } \\
\# of problems & $\begin{array}{c}\text { Police } \\
n=61)\end{array}$ & $\begin{array}{c}\text { Parents } \\
(n=359)\end{array}$ & $\begin{array}{c}\text { School } \\
(n=245)\end{array}$ & $\begin{array}{c}\text { Friends } \\
(n=161)\end{array}$ & $\begin{array}{c}\text { Any Problem } \\
(n=584)\end{array}$ \\
\hline 1 & $36 \%$ & $61 \%$ & $38 \%$ & $36 \%$ & $68 \%$ \\
2 & $38 \%$ & $24 \%$ & $39 \%$ & $33 \%$ & $22 \%$ \\
3 & $13 \%$ & $13 \%$ & $20 \%$ & $26 \%$ & $8 \%$ \\
4 & $13 \%$ & $2 \%$ & $3 \%$ & $5 \%$ & $2 \%$ \\
\hline
\end{tabular}

Table 9: Percentage of subjects with problems with police, parents, school and friends that reported only this one, 2, 3 or all 4 problems. 


\begin{tabular}{lcc}
\hline \multicolumn{3}{c}{ Obtainability of Drugs by Cannabis Intake } \\
Variable & $\begin{array}{c}\text { No Cannabis Use } \\
(n=3191)\end{array}$ & $\begin{array}{c}\text { Cannabis Use } \\
(n=973)\end{array}$ \\
\hline obtain amphetamine & 0.31 & 0.62 \\
obtain cocaine & 0.23 & 0.57 \\
obtain heroin & 0.19 & 0.47 \\
obtain ecstasy & 0.29 & 0.48 \\
\hline
\end{tabular}

Table 10: Means of obtain indicators (1 indicates that subjects can obtain the drug within 3 days) for various hard drugs in the data by cannabis intake.

\begin{tabular}{|c|c|c|c|c|}
\hline \multicolumn{5}{|c|}{ Cannabis and Hard Drug Use under different Frequency Cut-offs } \\
\hline \multicolumn{2}{|c|}{ User Definitions } & No Cannabis Use & Cannabis Use only & Hard Drug Use \\
\hline Cannabis & Hard Drugs & & & \\
\hline freq $_{c} \geq 1$ & freq $_{h} \geq 1$ & 2548 & 1045 & 571 \\
\hline $\operatorname{freq}_{c} \geq 5$ & freq $_{h} \geq 1$ & 3089 & 504 & 696 \\
\hline $\operatorname{freq}_{c} \geq 5$ & freq $_{h} \geq 5$ & 3160 & 661 & 343 \\
\hline
\end{tabular}

Table 11: Drug Use in the sample based on different frequency of use definitions. The second definition is the one used in the main empirical section, while the one in line 1 refers to Specification 1 in the sensitivity section and line 3 to Specification 2 in the sensitivity section.

\begin{tabular}{|c|c|c|c|}
\hline \multicolumn{4}{|c|}{ Extended Gateway Model with ignorant priors } \\
\hline & \multicolumn{2}{|c|}{ Hard uptake } & \multirow[t]{2}{*}{ Can uptake } \\
\hline & no can & can & \\
\hline Country & $0.03(0.18)$ & $0.37(0.18)$ & $-0.29(0.10)$ \\
\hline Oslo & $0.58(0.26)$ & $-1.11(0.46)$ & $-0.02(0.11)$ \\
\hline Obtain & $0.63(0.98)$ & $0.23(1.06)$ & $0.06(0.71)$ \\
\hline Seizure & $-0.37(0.64)$ & $3.21(1.11)$ & - \\
\hline Prevalence & $0.43(1.30)$ & $2.53(1.97)$ & $2.09(0.90)$ \\
\hline Non-Western & $-0.31(0.19)$ & $0.48(0.29)$ & $-0.54(0.13)$ \\
\hline Amphetamine Price & $0.36(0.41)$ & $0.04(0.44)$ & - \\
\hline Cannabis Price & - & - & $-0.75(1.38)$ \\
\hline Cannabis Use & 0.93 & $0.54)$ & - \\
\hline Police prob. & 0.88 & $0.22)$ & $0.61(0.19)$ \\
\hline Parents prob. & 0.46 & $0.10)$ & $0.42(0.08)$ \\
\hline School prob. & 0.03 & $0.13)$ & $0.25(0.11)$ \\
\hline Friends prob. & -0.04 & $(0.16)$ & $-0.09(0.13)$ \\
\hline Leave School early & 0.51 & $0.12)$ & $0.18(0.11)$ \\
\hline Alcohol young & 0.29 & $0.11)$ & $0.40(0.09)$ \\
\hline Smoker & 0.44 & $0.16)$ & $1.17(0.06)$ \\
\hline Male & 0.29 & $0.07)$ & $0.43(0.05)$ \\
\hline Age2124 & -0.02 & $(0.10)$ & $0.02(0.06)$ \\
\hline Intercept & -2.70 & $(0.47)$ & $-2.16(0.39)$ \\
\hline Correlation & $0.37(0.24)$ & $0.08(0.21)$ & \\
\hline
\end{tabular}

Table 12: Posterior means and standard deviations (parentheses) of the model parameters from the prior posterior analysis of the extended gateway model under the default prior. 
Frequencies of Hard Drug Use and Cannabis Use

Frequencies All All but Ecst Amph Coc Amph and Coc Ecs Her Can

\begin{tabular}{lcccccccc}
\hline$\geq 1$ & 571 & 534 & 419 & 419 & 535 & 295 & 63 & 1588 \\
$\geq 5$ & 343 & 329 & 270 & 237 & 330 & 155 & 32 & 971 \\
$\geq 11$ & 252 & 245 & 200 & 163 & 247 & 107 & 19 & 709 \\
$\geq 26$ & 178 & 171 & 142 & 106 & 171 & 73 & 15 & 505 \\
$\geq 50$ & 126 & 124 & 107 & 62 & 123 & 50 & 11 & 378 \\
\hline
\end{tabular}

Table 13: Frequency of hard drug use for various hard drugs and for cannabis.

\begin{tabular}{|c|c|c|c|c|c|c|}
\hline & \multicolumn{3}{|c|}{ All - Ecstasy } & \multicolumn{3}{|c|}{ Amphetamine } \\
\hline & \multicolumn{2}{|c|}{ Hard uptake } & \multirow[t]{2}{*}{ Can uptake } & \multicolumn{2}{|c|}{ Hard uptake } & \multirow[t]{2}{*}{ Can uptake } \\
\hline & no can & can & & no can & can & \\
\hline Country & $0.02(0.20)$ & $0.42(0.18)$ & $-0.27(0.10)$ & $-0.10(0.02)$ & $0.33(0.18)$ & $-0.26(0.10)$ \\
\hline Oslo & $0.53(0.29)$ & $-1.10(0.47)$ & $-0.03(0.11)$ & $0.11(0.35)$ & $-0.79(0.41)$ & $0.00(0.11)$ \\
\hline Obtain & $0.66(1.00)$ & $0.06(1.08)$ & $0.19(0.71)$ & $0.12(1.04)$ & $0.28(1.08)$ & $0.23(0.72)$ \\
\hline Seizure & $-0.09(0.69)$ & $3.22(1.13)$ & & $0.38(0.78)$ & $2.07(1.02)$ & \\
\hline Prevalence & $-0.27(1.34)$ & $2.40(1.98)$ & $2.19(0.90)$ & $-0.14(1.34)$ & $2.67(1.97)$ & $2.12(0.90)$ \\
\hline Non-Western & $-0.52(0.22)$ & $0.38(0.28)$ & $-0.50(0.13)$ & $-0.66(0.28)$ & $-0.04(0.27)$ & $-0.51(0.13)$ \\
\hline Amphetamine Price & $0.59(0.44)$ & $0.29(0.46)$ & - & $0.69(0.47)$ & $0.33(0.47)$ & - \\
\hline Cannabis Price & - & - & $-0.63(1.38)$ & - & - & $-0.57(1.38)$ \\
\hline Cannabis Use & \multicolumn{2}{|c|}{$1.18(0.55)$} & - & \multicolumn{2}{|c|}{$0.79(0.53)$} & - \\
\hline Police prob. & \multicolumn{2}{|c|}{$0.90(0.22)$} & $0.60(0.19)$ & \multicolumn{2}{|c|}{$0.86(0.21)$} & $0.59(0.19)$ \\
\hline Parents prob. & \multicolumn{2}{|c|}{$0.44(0.11)$} & $0.42(0.08)$ & \multicolumn{2}{|c|}{$0.39(0.11)$} & $0.41(0.08)$ \\
\hline School prob. & \multirow{2}{*}{\multicolumn{2}{|c|}{$0.04(0.13)$}} & $0.25(0.11)$ & \multicolumn{2}{|c|}{$0.08(0.13)$} & $0.24(0.11)$ \\
\hline Friends prob. & & & $-0.09(0.13)$ & \multicolumn{2}{|c|}{$-0.18(0.17)$} & $-0.08(0.13)$ \\
\hline Leave School early & \multicolumn{2}{|c|}{$0.56(0.12)$} & $0.19(0.11)$ & \multicolumn{2}{|c|}{$0.60(0.13)$} & $0.19(0.11)$ \\
\hline Alcohol young & \multirow{2}{*}{\multicolumn{2}{|c|}{$\begin{array}{l}0.30(0.11) \\
0.37(0.16)\end{array}$}} & $0.40(0.09)$ & \multirow{2}{*}{\multicolumn{2}{|c|}{$\begin{array}{l}0.39(0.11) \\
0.40(0.15)\end{array}$}} & $0.43(0.09)$ \\
\hline Smoker & & & $1.17(0.06)$ & & & $1.17(0.06)$ \\
\hline Male & \multicolumn{2}{|c|}{$0.29(0.08)$} & $0.42(0.05)$ & \multicolumn{2}{|c|}{$0.26(0.08)$} & $0.42(0.05)$ \\
\hline Age2124 & \multicolumn{2}{|c|}{$0.00(0.10)$} & $0.02(0.06)$ & \multicolumn{2}{|c|}{$-0.03(0.11)$} & $0.03(0.06)$ \\
\hline Intercept & \multicolumn{2}{|c|}{$-2.95(0.49)$} & $-2.27(0.39)$ & -2.72 & $(0.51)$ & $-2.29(0.39)$ \\
\hline Correlation & $0.28(0.26)$ & $0.02(0.21)$ & & $0.40(0.19)$ & $0.04(0.19)$ & \\
\hline
\end{tabular}

Table 14: Posterior means and standard deviations (parentheses) for parameters in the cannabis and hard drug equation from the prior posterior analysis of the extended gateway model for the new hard rug use variable that excludes ecstasy use and for amphetamine use only. 


\section{University Library}

\section{- M M N E R VA A gateway to Melbourne's research publications}

Minerva Access is the Institutional Repository of The University of Melbourne

Author/s:

Bretteville-Jensen, AL;Jacobi, L

Title:

CLIMBING THE DRUG STAIRCASE: A BAYESIAN ANALYSIS OF THE INITIATION OF HARD DRUG USE

Date:

2011-11-01

Citation:

Bretteville-Jensen, A. L. \& Jacobi, L. (2011). CLIMBING THE DRUG STAIRCASE: A BAYESIAN ANALYSIS OF THE INITIATION OF HARD DRUG USE. JOURNAL OF APPLIED ECONOMETRICS, 26 (7), pp.1157-1186. https://doi.org/10.1002/jae.1160.

Persistent Link:

http://hdl.handle.net/11343/122213 